\title{
On a Comparative Study of an Accurate Spatial Discretization Method for One-dimensional Continuous Systems
}

\author{
K. Wu, W.D. Zhu*, W. Fan \\ Department of Mechanical Engineering \\ University of Maryland, Baltimore County \\ 1000 Hilltop Circle, Baltimore, MD 21250, USA
}

\begin{abstract}
This work provides an in-depth investigation on advantages of a recently developed, new global spatial discretization method over the assumed modes method, and a clear description of the procedure and validity of the new method and its feasibility for arbitrary boundary conditions. A general formulation of the new spatial discretization method is given for second- and fourth-order continuous systems, whose displacements are divided into internal terms and boundary-induced terms, and two examples that consider the longitudinal vibration of a rod and the transverse vibration of a tensioned Euler-Bernoulli beam are used to demonstrate the new spatial discretization method. In the two examples, natural frequencies, mode shapes, harmonic steady-state responses, and transient responses of the systems are calculated using the new spatial discretization method and the assumed modes method, and results are compared with those from exact analyses. Convergence of the new spatial discretization method is investigated using different sets of trial functions for internal and boundary-induced terms. While the new spatial discretization method has additional degrees of freedom at boundaries of a continuous system compared with other global spatial discretization methods, it has the following advantages: (1) compared with the assumed modes method, the new method gives better results in calculating eigensolutions and responses of the system, and allows more terms to be retained in the trial function expansions due to the slowly growing condition number of the mass matrix of the system; and (2) compared with the exact eigenfunction expansion method, the new method can use sinusoidal functions as trial functions for the internal term rather than complicated eigenfunctions of the system.
\end{abstract}

Keywords: spatial discretization methods, assumed modes method, eigenfunction expansion

\footnotetext{
*Corresponding author

Email addresses: wukai1@umbc.edu (K. Wu), wzhu@umbc.edu (W.D. Zhu), fanwei@umbc.edu (W. Fan)
} 
method, internal term, boundary-induced term, convergence

\section{Nomenclature}

$\alpha, \beta, \gamma$ prescribed coefficients of a partial differential equation

$\mathbf{F}^{\mathbf{r}} \quad$ force vector of a second-order system

$\mathbf{F}^{\text {tb }}$ force vector of a fourth-order system

$\mathbf{K}^{\mathrm{r}} \quad$ stiffness matrix of a second-order system

$\mathbf{K}^{\text {tb }} \quad$ stiffness matrix of a fourth-order system

$\mathbf{M}^{\mathrm{r}} \quad$ mass matrix of a second-order system

$\mathbf{M}^{\text {tb }}$ mass matrix of a fourth-order system

q vector of generalized coordinates

$\Phi \quad$ vector of eigenfunctions

$\phi \quad$ vector of trial functions

$\hat{u} \quad$ boundary-induced term

$\lambda_{j} \quad j$-th eigenvalue

$\omega_{F} \quad$ frequency of the external harmonic force and moment

$\omega_{j} \quad j$-th natural frequency

$\Phi_{j} \quad j$-th eigenfunction

$\phi_{j} \quad j$-th trial function

$\rho \quad$ linear density of a continuous system

$\theta_{i} \quad i$-th interpolation function for boundary degrees of freedom

$\tilde{u} \quad$ internal term 
$\varphi_{j} \quad j$-th trial function for the internal term

$\xi, \eta \quad$ spatial and temporal steps in the finite difference method

$A_{0} \quad$ amplitude of the steady-state displacement at $x=l$

c damping coefficient

$e_{i} \quad i$-th boundary degree of freedom

$E A \quad$ axial stiffness of a second-order system

EI bending stiffness of a second-order system

F external force

$F_{0} \quad$ amplitude of the external harmonic force

$I, J \quad$ numbers of spatial and temporal nodes in the finite difference method

$I_{m} \quad$ rotatory inertia of a lumped sub-system

$K \quad$ number of additional boundary degrees of freedom

$k \quad$ stiffness of a lumped sub-system

$l \quad$ length of a continuous system

$M \quad$ external moment

$m \quad$ mass of a lumped sub-system

$M_{0} \quad$ amplitude of the external harmonic moment

$N \quad$ number of truncated terms

$P \quad$ tension in a continuous system

$p_{j} \quad j$-th generalized coordinate for the internal term

$q_{j} \quad j$-th generalized coordinate

$t \quad$ independent temporal variable 
u dependent variable

$x \quad$ independent spatial variable

$x_{1}, x_{2}$ boundary locations

\section{Introduction}

A vibrating system is usually studied using either a discrete or continuous system model [1]. A discrete system or lumped-parameter model has a finite number of degrees of freedom and a set of ordinary differential equations (ODEs) as its governing equations. A continuous system or distributed-parameter model has an infinite number of degrees of freedom and one or more partial differential equations (PDEs) as its governing equations, whose independent variables include spatial and temporal variables. Dynamic response of a discrete system is directly calculated using an ODE solver, and only displacements of the system and their time derivatives are obtained. Proper spatial discretization methods are needed for a continuous system so that its governing PDEs can be converted to a set of ODEs and dealt with using an ODE solver. Not only displacements of the system, but also their spatial derivatives, are of interest, since they are directly related to internal forces and moments of the system $[2,3]$. There are in general two classes of spatial discretization methods: local and global methods. The local methods, such as finite difference and finite element methods $[4,5]$, discretize a spatial domain into multiple smaller domains, and evaluate governing equations at connecting points between them. The finite element method can be combined with the transfer-matrix method to deal with wave propagation in a periodic structure [6]. The global methods, such as Trefftz, assumed modes, and Galerkin's methods, discretize a spatial domain using a set of trial functions. Trefftz method approximates a solution by a superposition of trial functions that satisfies a governing equation, with its unknown coefficients determined to satisfy boundary conditions [7]. Wave-based method is an indirect Trefftz method that can be used to calculate natural frequencies and mode shapes of a system [8]. The assumed modes method uses admissible functions that satisfy only geometric boundary conditions of a system as its trial functions, and Galerkin's method uses comparison functions that satisfy both geometric and natural boundary conditions as its trial functions $[1,9]$. Exact analysis can be performed on a system using the eigenfunction expansion method with trial functions in Galerkin's method being eigenfunctions of the system. However, Galerkin's method and exact analysis are not always feasible for systems 
with complicated boundary conditions, since comparison functions and eigenfunctions that satisfy these boundary conditions cannot be easily determined. Unlike the assumed modes and Galerkin's methods that can deal with initial boundary value problems of a continuous system, Rayleigh-Ritz method is based on stationarity of Rayleighs quotient and deals with eigenvalue problems of the system; it cannot be used to determine its dynamic response.

A new global spatial discretization method that can ensure all internal and boundary conditions of one-dimensional continuous systems are satisfied is recently developed [2] and used to calculate displacements and their spatial derivatives of moving elevator cable-car systems, the latter of which are related to tensions, bending moments, and shear forces in the cables [3]. The new spatial discretization method discretizes a continuous system with complicated boundary conditions by separating a displacement of the system into an internal term and a boundary-induced term $[2,3]$, where the internal term satisfies some predetermined homogeneous boundary conditions and the boundary-induced term accounts for corresponding boundary conditions that are not satisfied by the internal term using additional degrees of freedom at boundaries of the system, whose number does not exceed the number of boundary conditions of the system. The methodology is applicable to both linear and nonlinear systems and uniform convergence of solutions is shown in Ref. [2]. The new spatial discretization method is an advancement over the assumed modes method, since all the boundary conditions of a continuous system can be satisfied and uniform convergence of solutions is achieved. Consequently, spatial derivatives of displacements, which are related to tensions in cables, rods, and beams, and bending moments and shear forces in beams, can be accurately calculated using a term by term differentiation approach in the new spatial discretization method. It also has an improvement over Galerkin's method for two reasons: (1) Galerkin's method cannot always be used when a continuous system has complicated boundary conditions, since comparison functions that satisfy all the boundary conditions of the system cannot always be found; and (2) one can always use sinusoidal functions as trial functions for an internal term for all boundary conditions of second- and fourth-order continuous systems in the new spatial discretization method, while Galerkin's method may need to use hyperbolic functions as comparison functions for fourth-order systems when boundary conditions are not simply-supported ones.

The objectives of the present study are to provide an in-depth investigation on advantages of the new spatial discretization method over the assumed modes method via some simpler onedimensional continuous systems than those in Refs. [2,3], and a clearer description of the procedure 
and validity of the new method and its feasibility for arbitrary boundary conditions. A general formulation of the new spatial discretization method and its implementation are given for a PDE with arbitrary boundary conditions. Two continuous system models that consider the longitudinal vibration of a rod and the transverse vibration of a tensioned Euler-Bernoulli beam are studied; natural frequencies, mode shapes, harmonic steady-state responses, and transient responses of the systems are calculated using the assumed modes method and the new spatial discretization method, and results are compared with those from exact analyses. It is shown that geometric boundary conditions must be imposed, and natural boundary conditions are included in spatially discretized equations of the systems. Besides exact analyses, four sets of trial functions are used and compared, where the first two correspond to the assumed modes method and the last two correspond to the new spatial discretization method. The finite difference method is also used to calculate transient responses of the systems and compared with global spatial discretization methods. Damping and nonlinearity are not included in Secs. 3 and 4 for comparison purposes since exact solutions can be obtained there. Damping is included in an example in Sec. 5 to demonstrate that the new spatial discretization method can also be used to accurately solve for responses of damped systems.

\section{General Formulation of the New Spatial Discretization Method}

A general PDE that governs many one-dimensional continuous systems is given by

$$
\alpha \frac{\partial^{2} u(x, t)}{\partial t^{2}}+\beta \frac{\partial^{2} u(x, t)}{\partial x^{2}}+\gamma \frac{\partial^{4} u(x, t)}{\partial x^{4}}=0, \quad x_{1}<x<x_{2}, t>0,
$$

where $x$ and $t$ are independent spatial and temporal variables, respectively, $u$ is the dependent variable that denotes certain physical quantity, $x_{1}$ and $x_{2}$ are boundary locations for the spatial variable, and $\alpha, \beta$, and $\gamma$ are prescribed coefficients. Equation (1) with appropriately chosen coefficients $\alpha, \beta$, and $\gamma$ can act as the governing equation of a system in such fields as mechanics, acoustics, and electromagnetics.

The first step in the exact eigenfunction expansion method is to use separation of variables to express $u(x, t)$ as

$$
u(x, t)=\Phi(x) T(t),
$$

where $\Phi$ and $T$ are functions of $x$ and $t$, respectively. Substituting Eq. (2) into Eq. (1) yields

$$
\alpha \Phi(x) T^{\prime \prime}(t)+\beta \Phi^{\prime \prime}(x) T(t)+\gamma \Phi^{\prime \prime \prime \prime}(x) T(t)=0,
$$


where a prime denotes differentiation with respect to an argument. Rewriting Eq. (3) by separating $\Phi$ and $T$ terms yields

$$
-\frac{T^{\prime \prime}(t)}{T(t)}=\frac{\gamma \Phi^{\prime \prime \prime \prime}(x)+\beta \Phi^{\prime \prime}(x)}{\alpha \Phi(x)}=\lambda,
$$

where $\lambda$ is the eigenvalue of the system, and the following two equations are subsequently obtained:

$$
\begin{aligned}
T^{\prime \prime}(t)+\lambda T(t) & =0 \\
\gamma \Phi^{\prime \prime \prime \prime}(x)+\beta \Phi^{\prime \prime}(x)-\lambda \alpha \Phi(x) & =0 .
\end{aligned}
$$

The next step is to use boundary conditions of the system in Eqs. (5) and (6) and solve for eigenvalues $\lambda_{j}(j=1,2, \cdots)$ and corresponding eigenfunctions $\Phi_{j}(x)$, so that $u(x, t)$ can be expanded as

$$
u(x, t)=\sum_{j=1}^{\infty} \phi_{j}(x) q_{j}(t),
$$

where $\phi_{j}(x)$ denotes the $j$-th trial function, which is chosen to be $\Phi_{j}(x)$ here, and $q_{j}(t)$ are corresponding generalized coordinates. If boundary conditions of the system are complicated and its eigenvalues and eigenfunctions cannot be easily determined, $u(x, t)$ is usually spatially discretized using the assumed modes method that has the same expansion form as in Eq. (7), with trial functions $\phi_{j}(x)$ being admissible functions that satisfy only geometric boundary conditions of the system.

In the new spatial discretization method, $u(x, t)$ is expressed as the sum of an internal term $\tilde{u}(x, t)$ and a boundary-induced term $\hat{u}(x, t)$ for arbitrary boundary conditions $[2,3]$ :

$$
u(x, t)=\tilde{u}(x, t)+\hat{u}(x, t) .
$$

The internal term $\tilde{u}(x, t)$ is expressed in an expansion form

$$
\tilde{u}(x, t)=\sum_{j=1}^{\infty} \varphi_{j}(x) p_{j}(t)
$$

where $\varphi_{j}(x)(j=1,2, \cdots)$ are eigenfunctions of a system with homogeneous boundary conditions and $p_{j}(t)$ are corresponding generalized coordinates. For a second-order system, i.e. $\gamma=0$ and $\beta \neq 0$, two homogeneous boundary conditions are given by

$$
\frac{\partial^{s_{1}} \tilde{u}\left(x_{1}, t\right)}{\partial x^{s_{1}}}=0, \frac{\partial^{s_{2}} \tilde{u}\left(x_{2}, t\right)}{\partial x^{s_{2}}}=0
$$


where $s_{1}$ and $s_{2}$ are either 0 or 1 . For a fourth-order system, i.e. $\gamma \neq 0$, four homogeneous boundary conditions are given by

$$
\frac{\partial^{s_{1}} \tilde{u}\left(x_{1}, t\right)}{\partial x^{s_{1}}}=0, \frac{\partial^{s_{2}} \tilde{u}\left(x_{1}, t\right)}{\partial x^{s_{2}}}=0, \frac{\partial^{s_{3}} \tilde{u}\left(x_{2}, t\right)}{\partial x^{s_{3}}}=0, \frac{\partial^{s_{4}} \tilde{u}\left(x_{2}, t\right)}{\partial x^{s_{4}}}=0
$$

where $s_{1}, s_{2}, s_{3}$, and $s_{4}$ are $0,1,2$, or $3, s_{1} \neq s_{2}$, and $s_{3} \neq s_{4}$. The boundary-induced term $\hat{u}(x, t)$ is interpolated from boundary degrees of freedom $e_{i}(t)(i=1, \cdots, K)$ that are the dependent variable and/or its spatial derivatives at the boundaries:

$$
\hat{u}(x, t)=\sum_{i=1}^{K} \theta_{i}(x) e_{i}(t),
$$

where $\theta_{i}(x)$ are corresponding interpolation functions. The boundary-induced term needs to satisfy the following conditions:

$$
\begin{aligned}
& \frac{\partial^{s_{i}} \hat{u}\left(x_{1}, t\right)}{\partial x^{s_{i}}}=\frac{\partial^{s_{i}} u\left(x_{1}, t\right)}{\partial x^{s_{i}}}, \quad i=1, \cdots, \frac{K}{2}, \\
& \frac{\partial^{s_{i}} \hat{u}\left(x_{2}, t\right)}{\partial x^{s_{i}}}=\frac{\partial^{s_{i}} u\left(x_{2}, t\right)}{\partial x^{s_{i}}}, \quad i=\frac{K}{2}+1, \cdots, K .
\end{aligned}
$$

For a second-order system, $K=2$, the corresponding $e_{i}(t)(i=1,2)$ are given by

$$
e_{1}(t)=\frac{\partial^{s_{1}} u\left(x_{1}, t\right)}{\partial x^{s_{1}}}, e_{2}(t)=\frac{\partial^{s_{2}} u\left(x_{2}, t\right)}{\partial x^{s_{2}}}
$$

and the interpolation functions should satisfy

$$
\begin{aligned}
& \frac{d^{s_{1}} \theta_{1}\left(x_{1}\right)}{d x^{s_{1}}}=1, \frac{d^{s_{2}} \theta_{1}\left(x_{2}\right)}{d x^{s_{2}}}=0, \\
& \frac{d^{s_{1}} \theta_{2}\left(x_{1}\right)}{d x^{s_{1}}}=0, \frac{d^{s_{2}} \theta_{2}\left(x_{2}\right)}{d x^{s_{2}}}=1 .
\end{aligned}
$$

For a fourth-order system, $K=4$, the corresponding $e_{i}(t)(i=1,2,3,4)$ are given by

$$
e_{1}(t)=\frac{\partial^{s_{1}} u\left(x_{1}, t\right)}{\partial x^{s_{1}}}, e_{2}(t)=\frac{\partial^{s_{2}} u\left(x_{1}, t\right)}{\partial x^{s_{2}}}, e_{3}(t)=\frac{\partial^{s_{3}} u\left(x_{2}, t\right)}{\partial x^{s_{3}}}, e_{4}(t)=\frac{\partial^{s_{4}} u\left(x_{2}, t\right)}{\partial x^{s_{4}}},
$$

and the interpolation functions should satisfy

$$
\begin{aligned}
& \frac{d^{s_{1}} \theta_{1}\left(x_{1}\right)}{d x^{s_{1}}}=1, \frac{d^{s_{2}} \theta_{1}\left(x_{1}\right)}{d x^{s_{2}}}=0, \frac{d^{s_{3}} \theta_{1}\left(x_{2}\right)}{d x^{s_{3}}}=0, \frac{d^{s_{4}} \theta_{1}\left(x_{2}\right)}{d x^{s_{4}}}=0 \\
& \frac{d^{s_{1}} \theta_{2}\left(x_{1}\right)}{d x^{s_{1}}}=0, \frac{d^{s_{2}} \theta_{2}\left(x_{1}\right)}{d x^{s_{2}}}=1, \frac{d^{s_{3}} \theta_{2}\left(x_{2}\right)}{d x^{s_{3}}}=0, \frac{d^{s_{4}} \theta_{2}\left(x_{2}\right)}{d x^{s_{4}}}=0 \\
& \frac{d^{s_{1}} \theta_{3}\left(x_{1}\right)}{d x^{s_{1}}}=0, \frac{d^{s_{2}} \theta_{3}\left(x_{1}\right)}{d x^{s_{2}}}=0, \frac{d^{s_{3}} \theta_{3}\left(x_{2}\right)}{d x^{s_{3}}}=1, \frac{d^{s_{4}} \theta_{3}\left(x_{2}\right)}{d x^{s_{4}}}=0
\end{aligned}
$$




$$
\frac{d^{s_{1}} \theta_{4}\left(x_{1}\right)}{d x^{s_{1}}}=0, \frac{d^{s_{2}} \theta_{4}\left(x_{1}\right)}{d x^{s_{2}}}=0, \frac{d^{s_{3}} \theta_{4}\left(x_{2}\right)}{d x^{s_{3}}}=0, \frac{d^{s_{4}} \theta_{4}\left(x_{2}\right)}{d x^{s_{4}}}=1 .
$$

Substituting Eqs. (9) and (12) into Eq. (8) yields the expansion form of $u(x, t)$ :

$$
u(x, t)=\sum_{j=1}^{\infty} \varphi_{j}(x) p_{j}(t)+\sum_{i=1}^{K} \theta_{i}(x) e_{i}(t) .
$$

While trial functions of the internal term $\varphi_{j}(x)$ satisfy only homogeneous boundary conditions, the $K$ additional degrees of freedom at the boundaries $e_{i}(t)$ ensure that the expansion in Eq. (23) is capable of satisfying arbitrary boundary conditions of the system. Unlike the assumed modes method whose trial functions are required to satisfy geometric boundary conditions, trial functions of the internal term in the new spatial discretization method can be freely chosen as long as the dependent variable and its spatial derivatives at the boundaries are correspondingly chosen, with geometric boundary conditions imposed as constraints to ensure their satisfaction. Natural boundary conditions of the system are considered in spatially discretized equations of the system, as shown in Secs. 3 and 4. The infinite expansions in Eqs. (7) and (23) are truncated to $N$ terms and Eq. (23) can be written as

$$
u(x, t)=\sum_{j=1}^{N+K} \phi_{j}(x) q_{j}(t)
$$

where

$$
q_{j}(t)=p_{j}(t), \phi_{j}(x)=\varphi_{j}(x), \quad j=1, \cdots, N
$$

and

$$
q_{N+i}(t)=e_{i}(t), \phi_{N+i}(x)=\theta_{i}(x), \quad i=1, \cdots, K .
$$

With $K$ additional degrees of freedom compared with the exact eigenfunction expansion method and the assumed modes method, the new spatial discretization method can satisfy all the boundary conditions of the system.

\section{Example of a Second-Order System: the Longitudinal Vibration of a Rod}

Consider the longitudinal vibration of a rod with length $l$, linear density $\rho$, and axial stiffness $E A$, as shown in Fig. 1. The longitudinal displacement of the rod at position $x$ and time $t$ is denoted by $u(x, t)$. The rod has a fixed left boundary, a lumped sub-system with mass $m$ and stiffness $k$ attached to its right boundary, and a time-varying external force $F(t)$ exerted on the lumped mass. 


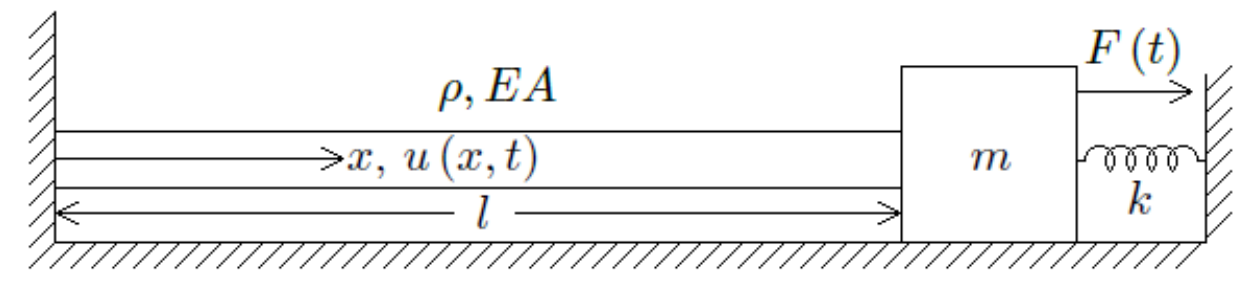

Figure 1: Schematic of a rod with a lumped sub-system attached to its right boundary

By setting $\alpha=\rho, \beta=-E A, \gamma=0, x_{1}=0$, and $x_{2}=l$ in Eq. (1), the governing equation of the system is

$$
\rho u_{t t}(x, t)-E A u_{x x}(x, t)=0, \quad 0<x<l, t>0,
$$

and corresponding boundary conditions are

$$
\begin{aligned}
u(0, t) & =0, \\
m u_{t t}(l, t)+k u(l, t)+E A u_{x}(l, t) & =F(t) .
\end{aligned}
$$

Note that Eq. (28) is a homogeneous geometric boundary condition and Eq. (29) is a complicated non-homogeneous natural boundary condition. The longitudinal displacement $u(x, t)$ can be expressed using Eq. (7) with the infinite series truncated to $N$ terms:

$$
u(x, t)=\sum_{j=1}^{N} \phi_{j}(x) q_{j}(t) .
$$

Substituting Eq. (30) into Eqs. (27)-(29) yields

$$
\begin{aligned}
\rho \sum_{j=1}^{N} \phi_{j}(x) q_{j}^{\prime \prime}(t)-E A \sum_{j=1}^{N} \phi_{j}^{\prime \prime}(x) q_{j}(t) & =0 \\
\sum_{j=1}^{N} \phi_{j}(0) q_{j}(t) & =0 \\
m \sum_{j=1}^{N} \phi_{j}(l) q_{j}^{\prime \prime}(t)+k \sum_{j=1}^{N} \phi_{j}(l) q_{j}(t) & \\
+E A \sum_{j=1}^{N} \phi_{j}^{\prime}(l) q_{j}(t) & =F(t) .
\end{aligned}
$$


By Eq. (32), trial functions $\phi_{j}(x)$ must satisfy

$$
\phi_{j}(0)=0, \quad j=1,2, \cdots, N \text {. }
$$

Multiplying Eq. (31) by $\phi_{i}(x)(i=1,2, \cdots, N)$, integrating it from 0 to $l$ and using integration by parts, and multiplying Eq. (33) by $\phi_{i}(l)$ yield

$$
\begin{aligned}
\sum_{j=1}^{N}\left[\int_{0}^{l} \rho \phi_{i}(x) \phi_{j}(x) d x\right] q_{j}^{\prime \prime}(t) & \\
+\sum_{j=1}^{N}\left[-\left.E A \phi_{i}(x) \phi_{j}^{\prime}(x)\right|_{0} ^{l}+\int_{0}^{l} E A \phi_{i}^{\prime}(x) \phi_{j}^{\prime}(x) d x\right] q_{j}(t) & =0, \\
\sum_{j=1}^{N}\left[m \phi_{i}(l) \phi_{j}(l)\right] q_{j}^{\prime \prime}(t)+\sum_{j=1}^{N}\left[k \phi_{i}(l) \phi_{j}(l)\right] q_{j}(t) & \\
+\sum_{j=1}^{N} E A \phi_{i}(l) \phi_{j}^{\prime}(l) q_{j}(t) & =\phi_{i}(l) F(t) .
\end{aligned}
$$

Spatially discretized equations of the system are obtained by adding Eqs. (35) and (36) and using Eq. (34):

$$
\begin{aligned}
& \sum_{j=1}^{N}\left[\int_{0}^{l} \rho \phi_{i}(x) \phi_{j}(x) d x+m \phi_{i}(l) \phi_{j}(l)\right] q_{j}^{\prime \prime}(t) \\
+ & \sum_{j=1}^{N}\left[\int_{0}^{l} E A \phi_{i}^{\prime}(x) \phi_{j}^{\prime}(x) d x+k \phi_{i}(l) \phi_{j}(l)\right] q_{j}(t)=\phi_{i}(l) F(t), \quad i=1,2, \cdots, N,
\end{aligned}
$$

which can be written in a matrix form

$$
\mathbf{M}^{r} \mathbf{q}^{\prime \prime}(t)+\mathbf{K}^{r} \mathbf{q}(t)=\mathbf{F}^{r}(t)
$$

where $\mathbf{q}(t)=\left[\begin{array}{llll}q_{1}(t) & q_{2}(t) & \cdots & q_{N}(t)\end{array}\right]^{T}$ is the vector of generalized coordinates,

$$
\mathbf{M}^{r}=\int_{0}^{l} \rho \phi(x) \phi(x)^{T} d x+m \boldsymbol{\phi}(l) \boldsymbol{\phi}(l)^{T}
$$

and

$$
\mathbf{K}^{r}=\int_{0}^{l} E A \boldsymbol{\phi}^{\prime}(x) \boldsymbol{\phi}^{\prime}(x)^{T} d x+k \boldsymbol{\phi}(l) \boldsymbol{\phi}(l)^{T}
$$

are $N \times N$ symmetric mass and stiffness matrices of the system, respectively, in which

$$
\phi(x)=\left[\begin{array}{llll}
\phi_{1}(x) & \phi_{2}(x) & \cdots & \phi_{N}(x)
\end{array}\right]^{T}
$$


is the vector of trial functions, and

$$
\mathbf{F}^{r}(t)=\phi(l) F(t)
$$

is the $N \times 1$ force vector of the system.

In the exact eigenvalue problem analysis, eigenfunctions of the system are obtained by solving the associated eigenvalue problem:

$$
\Phi_{j}(x)=\sin \left(\sqrt{\frac{\rho}{E A}} \omega_{j} x\right), \quad j=1,2, \cdots,
$$

where $\omega_{j}$ are natural frequencies of the system obtained from the frequency equation

$$
\left(k-m \omega_{j}^{2}\right) \sin \left(\sqrt{\frac{\rho l^{2}}{E A}} \omega_{j}\right)+\sqrt{\rho E A} \omega_{j} \cos \left(\sqrt{\frac{\rho l^{2}}{E A}} \omega_{j}\right)=0 .
$$

The exact eigenfunction expansion method uses eigenfunctions of the system as trial functions in Eq. (30), and its results can be used as benchmarks to evaluate performances of the assumed modes method and the new spatial discretization method with two different sets of trial functions chosen for each method. The first set of trial functions, which is used in the assumed modes method, is chosen as eigenfunctions of a fixed-free rod

$$
\phi_{j}(x)=\sin \left(\left(j-\frac{1}{2}\right) \pi \frac{x}{l}\right), \quad j=1,2, \cdots, N
$$

which satisfy only the geometric boundary condition in Eq. (28). The second set of trial functions, which is also used in the assumed modes method, is chosen as a set of quasi-comparison functions $[1,9]$

$$
\phi_{j}(x)=\sin \left(\frac{j \pi x}{2 l}\right), \quad j=1,2, \cdots, N,
$$

which satisfy the geometric boundary condition in Eq. (28) and is capable of satisfying the natural boundary condition in Eq. (29). Note that $\phi_{j}(x)$ are eigenfunctions of a fixed-free rod when $j$ are odd integers, and they are eigenfunctions of a fixed-fixed rod when $j$ are even integers. In the new spatial discretization method, only one boundary degree of freedom is required at $x=l$ since the boundary condition in Eq. (28) is a homogeneous geometric boundary condition. With the displacement $e_{2}(t)=u(l, t)$ chosen as the boundary degree of freedom, the third set of trial functions, which is used in the new spatial discretization method, is chosen as

$$
\begin{aligned}
\phi_{j}(x)=\varphi_{j}(x) & =\sin \left(j \pi \frac{x}{l}\right), \quad j=1,2, \cdots, N, \\
\phi_{N+1}(x)=\theta_{2}(x) & =\frac{x}{l},
\end{aligned}
$$


where $\varphi_{j}(x)$ are eigenfunctions of a fixed-fixed rod and $\theta_{2}(x)$ is a polynomial that satisfies Eq. (17). With the first spatial derivative of the displacement $e_{2}(t)=u_{x}(l, t)$ chosen as the boundary degree of freedom, the fourth set of trial functions, which is also used in the new spatial discretization method, is chosen as

$$
\begin{aligned}
\phi_{j}(x) & =\varphi_{j}(x)=\sin \left(\left(j-\frac{1}{2}\right) \pi \frac{x}{l}\right), \quad j=1,2, \cdots, N, \\
\phi_{N+1}(x) & =\theta_{2}(x)=x,
\end{aligned}
$$

where $\varphi_{j}(x)$ are eigenfunctions of a fixed-free rod and $\theta_{2}(x)$ is a polynomial that satisfies Eq. (17).

\subsection{Natural frequencies and mode shapes}

Parameters of the rod are given by $l=1, \rho=1, E A=1, m=1$, and $k=1$. Natural frequencies and mode shapes of the system are calculated by solving the eigenvalue problem

$$
\left(\mathbf{K}^{r}-\mathbf{M}^{r} \omega^{2}\right) \boldsymbol{\Phi}=\mathbf{0}
$$

using the four sets of trial functions, where $\omega$ is the natural frequency and

$$
\boldsymbol{\Phi}=\left[\begin{array}{llll}
\Phi_{1}(x) & \Phi_{2}(x) & \cdots & \Phi_{N}(x)
\end{array}\right]^{T}
$$

is the vector of eigenfunctions. Natural frequencies obtained from the four sets of trial functions when $N=10$ are compared with those from the exact eigenvalue problem analysis, and relative errors are shown in Fig. 2. Natural frequencies from the first set of trial functions with the assumed modes method have visible errors for the second through tenth frequencies; those from the second set with the assumed modes method have small errors for the first seven frequencies, but the errors drastically increase for the last three frequencies. Since the second set of trial functions is not a set of orthogonal eigenfunctions of a simpler self-adjoint system, the corresponding mass matrix $\mathbf{M}^{r}$ is not diagonal anymore, which leads to rapidly increasing condition number of the mass matrix and large numerical error when $N$ is large. Natural frequencies from the third and fourth sets of trial functions with the new spatial discretization method have overall small errors, and the third set performs better than the fourth one. While mass matrices from the third and fourth sets of trial functions are not diagonal, their first $N \times N$ sub-matrices are diagonal and non-zero off-diagonal entries of the matrices appear only in the last $K$ rows and columns, which makes their condition numbers grow much more slowly than that from the second set of trial functions, and $N$ can be chosen as a relatively large number without compromising the calculation. Note that in numerical 
implementation, with use of some algebraic manipulation, the inverse of the mass matrix can be obtained by dividing it into four sub-matrices and calculating the inverse of a $K \times K$ matrix with $K \leq 4$. The first, second, ninth, and tenth mode shapes calculated from the four sets of trial functions are compared with those from the exact eigenvalue problem analysis, as shown in Fig. 3, and only the third and fourth sets with the new spatial discretization method can give accurate results.

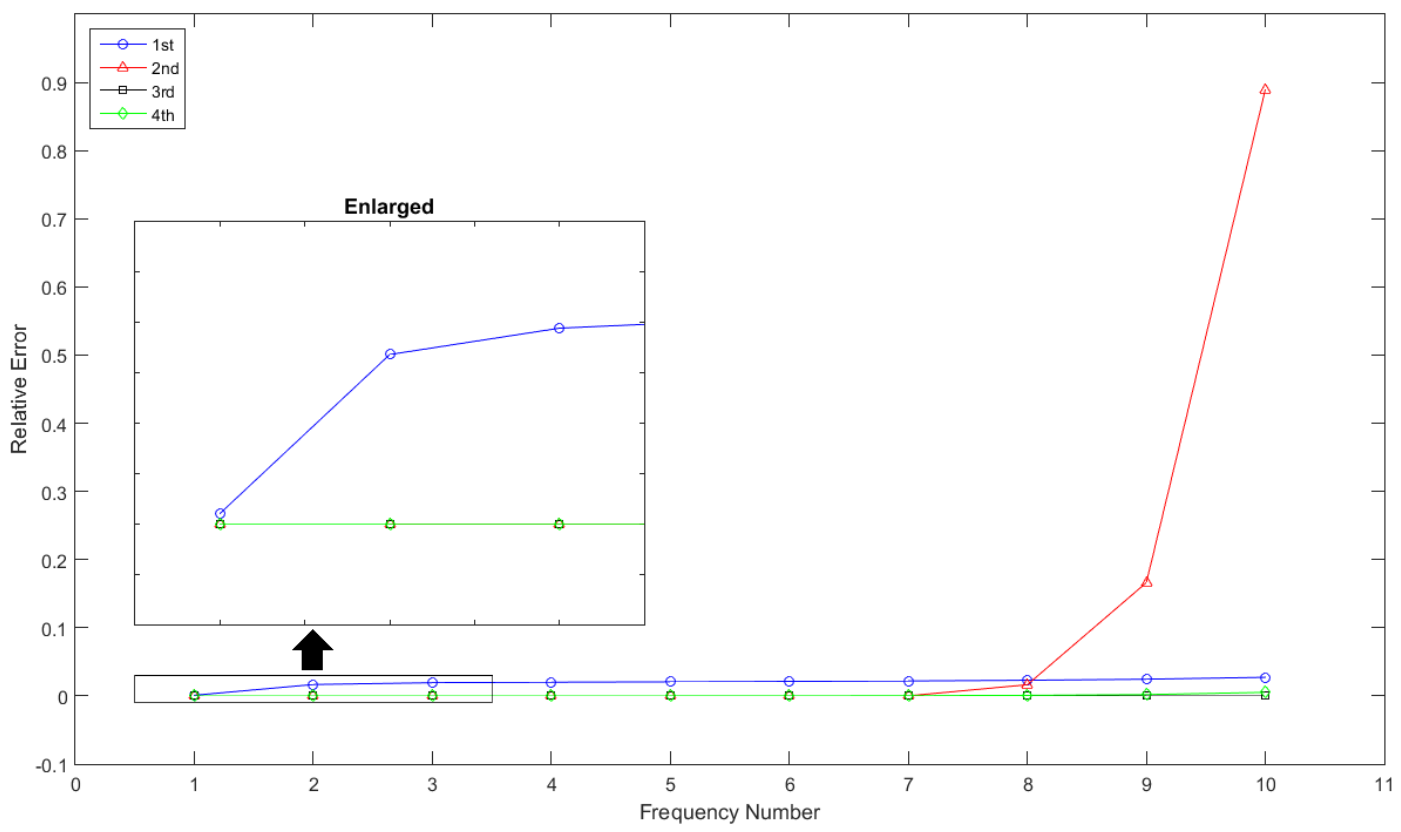

Figure 2: Relative errors of the first 10 natural frequencies of the $\operatorname{rod}$ when $N=10$, where the " $\bigcirc$ ", " $\triangle$ ", " $\square$ ", and " $\diamond$ " signs correspond to the first, second, third, and fourth sets of trial functions, respectively. 

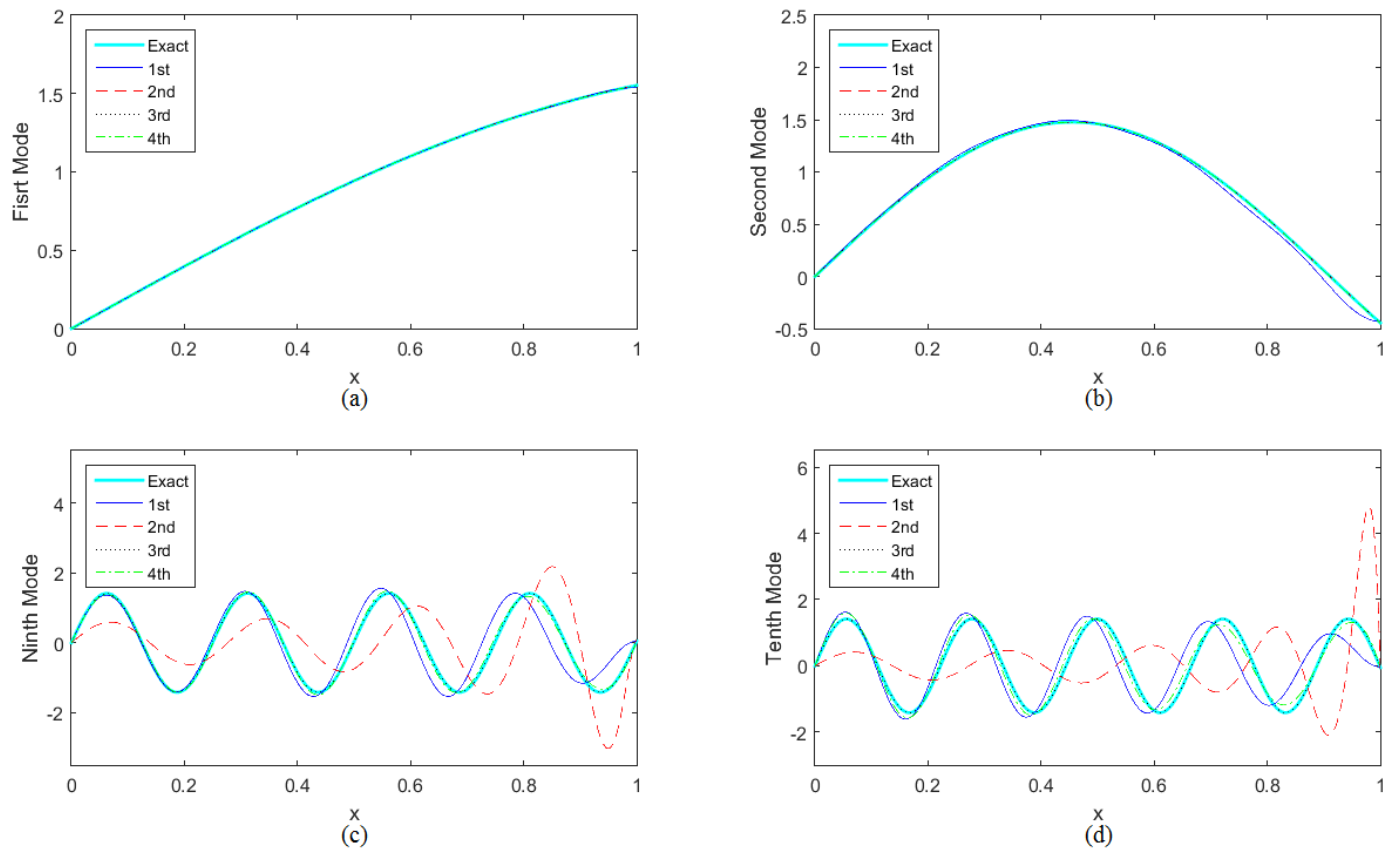

Figure 3: (a) First, (b) second, (c) ninth, and (d) tenth mode shapes of the rod when $N=10$, where solid, dashed, dotted, and dash-dotted lines correspond to the first, second, third, and fourth sets of trial functions, respectively, and thick solid lines correspond to the exact eigenvalue problem analysis.

\subsection{Harmonic steady-state response}

Parameters of the rod are the same as those in Sec. 3.1; the external force is $F(t)=F_{0} \sin \left(\omega_{F} t\right)$, where $\omega_{F}$ is the excitation frequency. In the exact harmonic steady-state response analysis, the displacement of the rod is assumed to be

$$
u(x, t)=U(x) \sin \left(\omega_{F} t\right) .
$$

Substituting Eq. (53) into Eqs. (27)-(29) yields

$$
\begin{aligned}
U^{\prime \prime}(x)+\frac{\rho}{E A} \omega_{F}^{2} U(x) & =0, \\
U(0) & =0, \\
\left(k-m \omega_{F}^{2}\right) U(l)+E A U^{\prime}(l) & =F_{0},
\end{aligned}
$$

whose solution is

$$
U(x)=U_{0} \sin \left(\sqrt{\frac{\rho}{E A}} \omega_{F} x\right),
$$


where

$$
U_{0}=\frac{F_{0}}{\left(k-m \omega_{F}^{2}\right) \sin \left(\sqrt{\frac{\rho l^{2}}{E A}} \omega_{F}\right)+\sqrt{\rho E A} \omega_{F} \cos \left(\sqrt{\frac{\rho l^{2}}{E A}} \omega_{F}\right)} .
$$

Note that the denominator on the right-hand side of Eq. (58) is the left-hand side of the frequency equation in Eq. (44) with $\omega_{j}$ replaced by $\omega_{F}$. The displacement of the rod at $x=l$ is given by

$$
\begin{aligned}
u(l, t)= & U(l) \sin \left(\omega_{F} t\right) \\
= & \frac{F_{0} \sin \left(\sqrt{\frac{\rho l^{2}}{E A}} \omega_{F}\right)}{\left(k-m \omega_{F}^{2}\right) \sin \left(\sqrt{\frac{\rho l^{2}}{E A}} \omega_{F}\right)+\sqrt{\rho E A} \omega_{F} \cos \left(\sqrt{\frac{\rho l^{2}}{E A}} \omega_{F}\right)} \sin \left(\omega_{F} t\right) .
\end{aligned}
$$

When $\omega_{F} \neq \omega_{j}(j=1,2, \cdots, N)$, its amplitude is

$$
A_{0}=\left|\frac{F_{0} \sin \left(\sqrt{\frac{\rho l^{2}}{E A}} \omega_{F}\right)}{\left(k-m \omega_{F}^{2}\right) \sin \left(\sqrt{\frac{\rho l^{2}}{E A}} \omega_{F}\right)+\sqrt{\rho E A} \omega_{F} \cos \left(\sqrt{\frac{\rho l^{2}}{E A}} \omega_{F}\right)}\right| .
$$

Note that the amplitude in Eq. (60) approaches infinity when $\omega_{F}$ approaches one of the natural frequencies of the system.

In a trial function expansion method, the generalized coordinate vector is assumed to be

$$
\mathbf{q}(t)=\mathbf{Q} \sin \left(\omega_{F} t\right)
$$

where $\mathbf{Q}$ is a constant vector. Substituting Eq. (61) into Eq. (38) yields

$$
\left(\mathbf{K}^{r}-\omega_{F}^{2} \mathbf{M}^{r}\right) \mathbf{Q}=F_{0} \phi(l),
$$

from which $\mathbf{Q}$ is obtained as

$$
\mathbf{Q}=F_{0}\left(\mathbf{K}^{r}-\omega_{F}^{2} \mathbf{M}^{r}\right)^{-1} \boldsymbol{\phi}(l)
$$

The displacement of the rod at $x=l$ is given by

$$
\begin{aligned}
u(l, t) & =\boldsymbol{\phi}(l)^{T} \mathbf{Q} \sin \left(\omega_{F} t\right) \\
& =F_{0} \boldsymbol{\phi}(l)^{T}\left(\mathbf{K}^{r}-\omega_{F}^{2} \mathbf{M}^{r}\right)^{-1} \boldsymbol{\phi}(l) \sin \left(\omega_{F} t\right),
\end{aligned}
$$

and its amplitude is

$$
A_{0}=\left|F_{0} \boldsymbol{\phi}(l)^{T}\left(\mathbf{K}^{r}-\omega_{F}^{2} \mathbf{M}^{r}\right)^{-1} \boldsymbol{\phi}(l)\right|, \quad \omega_{F} \neq \omega_{j}
$$


where $j=1,2, \cdots, N$.

Amplitudes of the displacement $u(l, t)$ with $F_{0}=1$ from the exact harmonic steady-state response analysis and the four sets of trial functions with $N=10$, which are amplitudes of the corresponding frequency response function, are shown in Fig. 4. The first and second sets of trial functions with the assumed modes method cannot accurately calculate the amplitudes, and amplitudes calculated from the third and fourth sets of trial functions with the new spatial discretization method match well with that obtained from the exact harmonic steady-state response analysis.

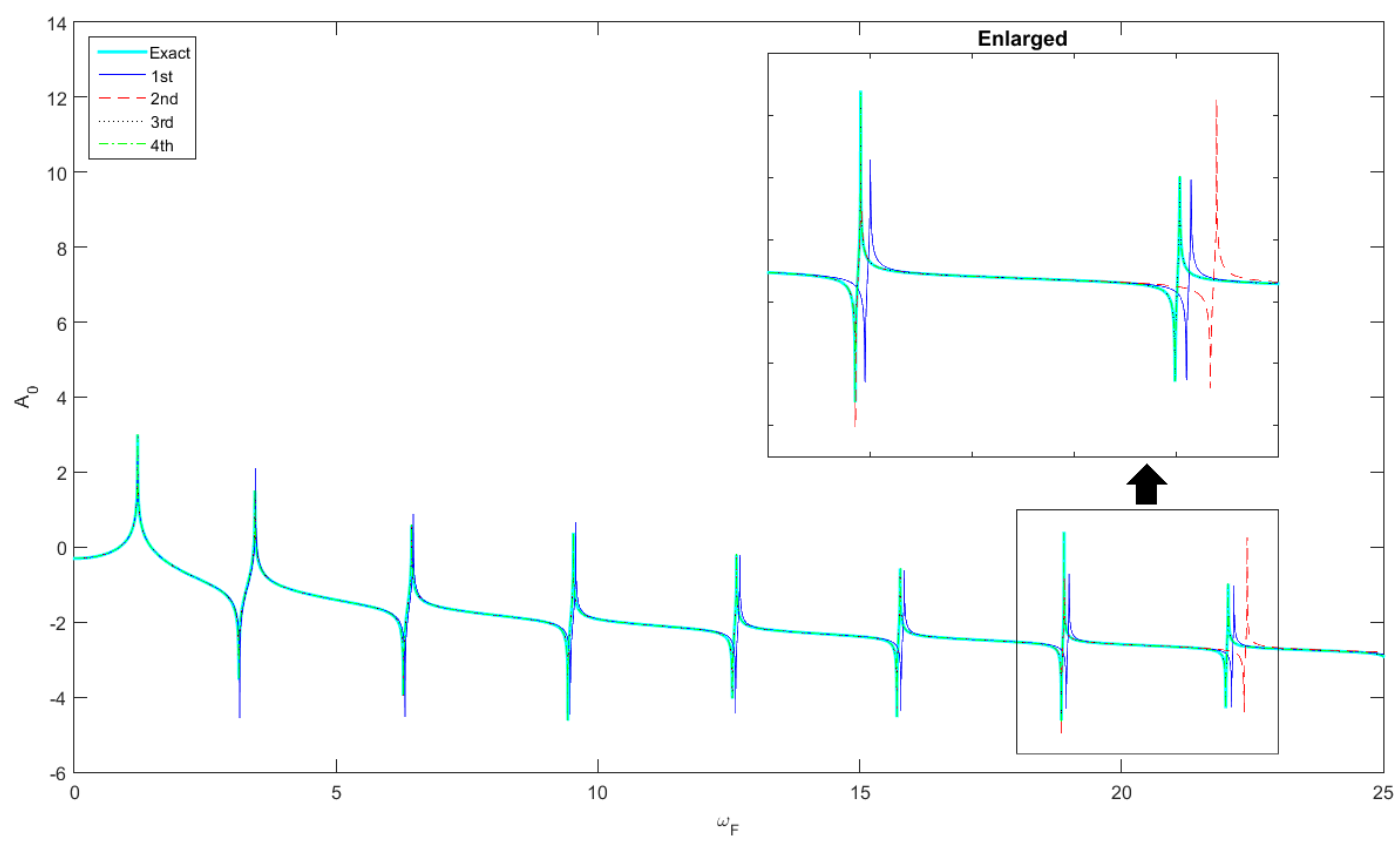

Figure 4: Amplitudes of $u(l, t)$ with varying excitation frequencies, where solid, dashed, dotted, and dash-dotted lines correspond to the first, second, third, and fourth sets of trial functions, respectively, and the thick solid line corresponds to the exact harmonic steady-state response analysis.

\subsection{Transient response}

Displacements and axial forces of the system at $t=5$ and at $x=l$, with zero initial conditions and $\omega_{F}=20$, are shown in Figs. 5 and 6, respectively; $N=40$ for the first, third, and fourth sets of trial functions, and $N=10$ for the second set of trial functions due to the corresponding ill-conditioned mass matrix. Note that axial forces are related to first spatial derivatives of 
the displacements. Results from the first and second sets of trial functions are not accurate, and those from the third and fourth sets compare well with those from the exact eigenfunction expansion method and the finite difference method shown in Appendix A with numbers of spatial and temporal nodes being $I=401$ and $J=60001$, respectively.
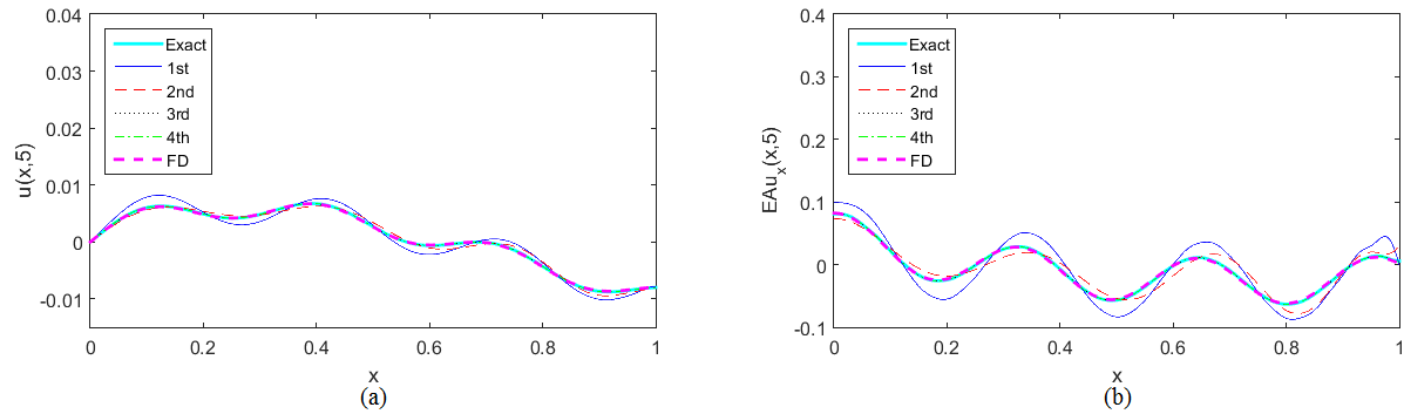

Figure 5: (a) Displacements and (b) axial forces of the rod at $t=5$, where solid, dashed, dotted, and dashdotted lines correspond to the first, second, third, and fourth sets of trial functions, respectively, thick solid lines correspond to the exact eigenfunction expansion method, and thick dashed lines correspond to the finite difference (FD) method.
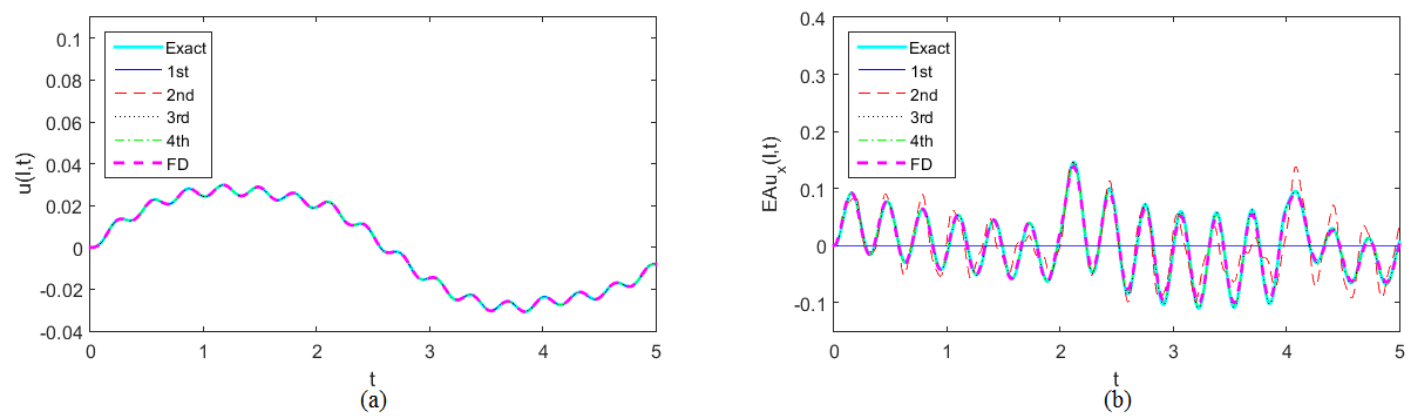

Figure 6: (a) Displacements and (b) axial forces of the rod at $x=l$, where solid, dashed, dotted, and dashdotted lines correspond to the first, second, third, and fourth sets of trial functions, respectively, thick solid lines correspond to the exact eigenfunction expansion method, and thick dashed lines correspond to the finite difference (FD) method. 


\section{Example of a Fourth-Order System: the Transverse Vibration of a Tensioned Euler- Bernoulli Beam}

Consider the transverse vibration of a tensioned Euler-Bernoulli beam with length $l$, linear density $\rho$, tension $P$, and bending stiffness $E I$, as shown in Fig. 7. The transverse displacement of the beam at position $x$ and time $t$ is denoted by $u(x, t)$. The beam has a clamped left boundary, a lumped sub-system with mass $m$, rotatory inertia $I_{m}$, and stiffness $k$ attached to its right boundary, and a time-varying external moment $M(t)$ and force $F(t)$ exerted on the lumped mass.

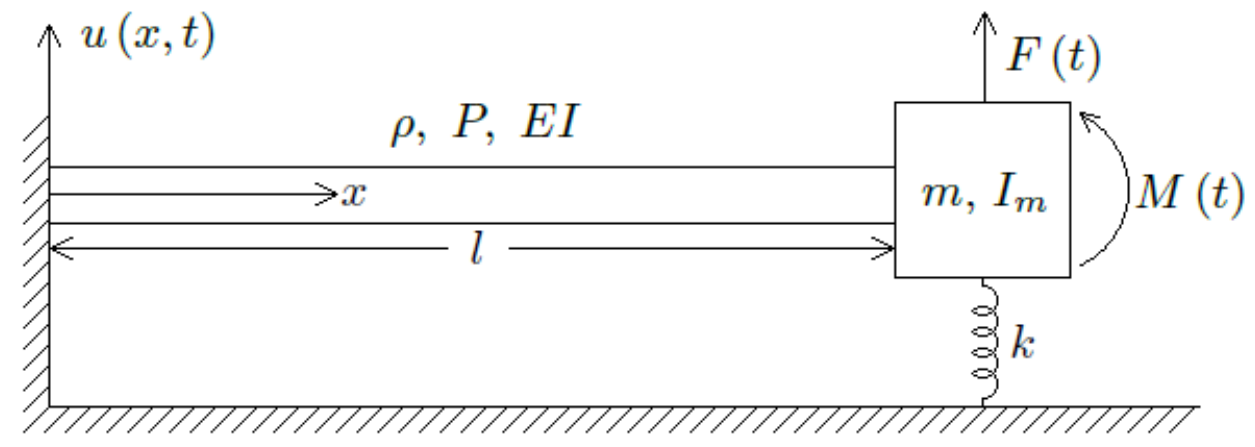

Figure 7: Schematic of a tensioned Euler-Bernoulli beam with a lumped sub-system attached to its right boundary

By setting $\alpha=\rho, \beta=-P, \gamma=E I, x_{1}=0$, and $x_{2}=l$ in Eq. (1), the governing equation of the system is

$$
\rho u_{t t}(x, t)-P u_{x x}(x, t)+E I u_{x x x x}(x, t)=0, \quad 0<x<l, t>0,
$$

and corresponding boundary conditions are

$$
\begin{aligned}
u(0, t) & =0, \\
u_{x}(0, t) & =0, \\
I_{m} u_{x t t}(l, t)+E I u_{x x}(l, t) & =M(t), \\
m u_{t t}(l, t)+k u(l, t)+P u_{x}(l, t)-E I u_{x x x}(l, t) & =F(t) .
\end{aligned}
$$

Note that Eqs. (67) and (68) are homogeneous geometric boundary conditions, and Eqs. (69) and (70) are complicated non-homogeneous natural boundary conditions. The transverse displacement 
$u(x, t)$ can be expressed by Eq. (30). Substituting Eq. (30) into Eqs. (66)-(70) yields

$$
\begin{aligned}
\rho \sum_{j=1}^{N} \phi_{j}(x) q_{j}^{\prime \prime}(t)-P \sum_{j=1}^{N} \phi_{j}^{\prime \prime}(x) q_{j}(t)+E I \sum_{j=1}^{N} \phi_{j}^{\prime \prime \prime \prime}(x) q_{j}(t) & =0 \\
\sum_{j=1}^{N} \phi_{j}(0) q_{j}(t) & =0 \\
\sum_{j=1}^{N} \phi_{j}^{\prime}(0) q_{j}(t) & =0 \\
I_{m} \sum_{j=1}^{N} \phi_{j}^{\prime}(l) q_{j}^{\prime \prime}(t)+E I \sum_{j=1}^{N} \phi_{j}^{\prime \prime}(l) q_{j}(t) & =M(t), \\
m \sum_{j=1}^{N} \phi_{j}(l) q_{j}^{\prime \prime}(t)+k \sum_{j=1}^{N} \phi_{j}(l) q_{j}(t)+P \sum_{j=1}^{N} \phi_{j}^{\prime}(l) q_{j}(t)-E I \sum_{j=1}^{N} \phi_{j}^{\prime \prime \prime}(l) q_{j}(t) & =F(t) .
\end{aligned}
$$

By Eqs. (72) and (73), trial functions $\phi_{j}(x)$ must satisfy

$$
\begin{aligned}
& \phi_{j}(0)=0, \quad j=1,2, \cdots, N, \\
& \phi_{j}^{\prime}(0)=0, \quad j=1,2, \cdots, N .
\end{aligned}
$$

Multiplying Eq. (71) by $\phi_{i}(x)(i=1,2, \cdots, N)$, integrating it from 0 to $l$ and using integration by parts, multiplying Eq. (74) by $\phi_{i}^{\prime}(l)$, and multiplying Eq. (75) by $\phi_{i}(l)$ yield

$$
\begin{aligned}
\sum_{j=1}^{N}\left[\int_{0}^{l} \rho \phi_{i}(x) \phi_{j}(x) d x\right] q_{j}^{\prime \prime}(t) & \\
\sum_{j=1}^{N}\left[-\left.P \phi_{i}(x) \phi_{j}^{\prime}(x)\right|_{0} ^{l}+P \int_{0}^{l} \phi_{i}^{\prime}(x) \phi_{j}^{\prime}(x) d x\right] q_{j}(t) & \\
+\sum_{j=1}^{N}\left[\left.E I \phi_{i}(x) \phi_{j}^{\prime \prime \prime}(x)\right|_{0} ^{l}-\left.E I \phi_{i}^{\prime}(x) \phi_{j}^{\prime \prime}(x)\right|_{0} ^{l}+\int_{0}^{l} E I \phi_{i}^{\prime \prime}(x) \phi_{j}^{\prime \prime}(x) d x\right] q_{j}(t) & =0, \\
\sum_{j=1}^{N}\left[I_{m} \phi_{i}^{\prime}(l) \phi_{j}^{\prime}(l)\right] q_{j}^{\prime \prime}(t)+\sum_{j=1}^{N}\left[E I \phi_{i}^{\prime}(l) \phi_{j}^{\prime \prime}(l)\right] q_{j}(t) & =\phi_{i}^{\prime}(l) M(t)(79) \\
+\sum_{j=1}^{N}\left[P \phi_{i}(l) \phi_{j}^{\prime}(l)\right] q_{j}(t)-\sum_{j=1}^{N}\left[E I \phi_{i}(l) \phi_{j}^{\prime \prime \prime}(l)\right] q_{j}(t) & =\phi_{i}(l) F(t) \cdot(80)
\end{aligned}
$$


Spatially discretized equations of the system are obtained by adding Eqs. (78)-(80), and using Eqs. (76) and (77):

$$
\begin{gathered}
\sum_{j=1}^{N}\left[\int_{0}^{l} \rho \phi_{i}(x) \phi_{j}(x) d x+m \phi_{i}(l) \phi_{j}(l)+I_{m} \phi_{i}^{\prime}(l) \phi_{j}^{\prime}(l)\right] q_{j}^{\prime \prime}(t) \\
+\sum_{j=1}^{N}\left[\int_{0}^{l} P \phi_{i}^{\prime}(x) \phi_{j}^{\prime}(x) d x+\int_{0}^{l} E I \phi_{i}^{\prime \prime}(x) \phi_{j}^{\prime \prime}(x) d x+k \phi_{i}(l) \phi_{j}(l)\right] q_{j}(t)=\phi_{i}^{\prime}(l) M(t) \\
+\phi_{i}(l) F(t)(81)
\end{gathered}
$$

which can be written in the matrix form

$$
\mathbf{M}^{t b} \mathbf{q}^{\prime \prime}(t)+\mathbf{K}^{t b} \mathbf{q}(t)=\mathbf{F}^{t b}(t)
$$

where

$$
\mathbf{M}^{t b}=\int_{0}^{l} \rho \boldsymbol{\phi}(x) \boldsymbol{\phi}(x)^{T} d x+m \boldsymbol{\phi}(l) \boldsymbol{\phi}(l)^{T}+I_{m} \boldsymbol{\phi}^{\prime}(l) \boldsymbol{\phi}^{\prime}(l)^{T}
$$

and

$$
\mathbf{K}^{t b}=\int_{0}^{l} P \boldsymbol{\phi}^{\prime}(x) \boldsymbol{\phi}^{\prime}(x)^{T} d x+\int_{0}^{l} E I \boldsymbol{\phi}^{\prime \prime}(x) \boldsymbol{\phi}^{\prime \prime}(x)^{T} d x+k \boldsymbol{\phi}(l) \boldsymbol{\phi}(l)^{T}
$$

are $N \times N$ symmetric mass and stiffness matrices of the system, respectively, and

$$
\mathbf{F}^{t b}(t)=\phi^{\prime}(l) M(t)+\phi(l) F(t)
$$

is the $N \times 1$ force vector of the system. 
In the exact eigenvalue problem analysis, eigenfunctions of the system are obtained by solving the associated eigenvalue problem:

$$
\begin{aligned}
\Phi_{j}(x)= & \cos \left(\beta_{1 j} x\right) \\
& -\sin \left(\beta_{1 j} x\right)\left\{I_{m} \omega_{j}^{2}\left[-2 \beta_{1 j} e^{-\beta_{2 j} l} \sin \left(\beta_{1 j} l\right)-\beta_{2 j}+\beta_{2 j} e^{-2 \beta_{2 j} l}\right]\right. \\
& \left.+E I\left[2 \beta_{1 j}^{2} e^{-\beta_{2 j} l} \cos \left(\beta_{1 j} l\right)+\beta_{2 j}^{2}+\beta_{2 j}^{2} e^{-2 \beta_{2 j} l}\right]\right\} \\
& \div\left\{I_{m} \omega_{j}^{2}\left[2 \beta_{1 j} e^{-\beta_{2 j} l} \cos \left(\beta_{1 j} l\right)-\beta_{1 j}-\beta_{1 j} e^{-2 \beta_{2 j} l}\right]\right. \\
& \left.+E I\left[2 \beta_{1 j}^{2} e^{-\beta_{2 j} l} \sin \left(\beta_{1 j} l\right)+\beta_{1 j} \beta_{2 j}-\beta_{1 j} \beta_{2 j} e^{-2 \beta_{2 j} l}\right]\right\} \\
& -\left[\left\{I_{m} \omega_{j}^{2} \beta_{1 j}\left[\cos \left(\beta_{1 j} l\right)+\frac{\beta_{1 j}}{\beta_{2 j}} \sin \left(\beta_{1 j} l\right)-e^{-\beta_{2 j} l}\right]\right.\right. \\
& \left.+E I \beta_{1 j}^{2}\left[\sin \left(\beta_{1 j} l\right)-\frac{\beta_{1 j}}{\beta_{2 j}} \cos \left(\beta_{1 j} l\right)-\frac{\beta_{2 j}}{\beta_{1 j}} e^{-\beta_{2 j} l}\right]\right\} e^{\beta_{2 j}(x-l)} \\
+ & \left(I_{m} \omega_{j}^{2} \beta_{1 j}\left\{e^{-\beta_{2 j} l}\left[\cos \left(\beta_{1 j} l\right)-\frac{\beta_{1 j}}{\beta_{2 j}} \sin \left(\beta_{1 j} l\right)\right]-1\right\}\right. \\
+ & \left.\left.+E I \beta_{1 j}^{2}\left\{e^{-\beta_{2 j} l}\left[\sin \left(\beta_{1 j} l\right)+\frac{\beta_{1 j}}{\beta_{2 j}} \cos \left(\beta_{1 j} l\right)\right]+\frac{\beta_{2 j}}{\beta_{1 j}}\right\}\right) e^{-\beta_{2 j} x}\right] \\
& \div\left\{I_{m} \omega_{j}^{2}\left[2 \beta_{1 j} e^{-\beta_{2 j} l} \cos \left(\beta_{1 j} l\right)-\beta_{1 j}-\beta_{1 j} e^{-2 \beta_{2 j} l}\right]\right. \\
+ & \left.E I\left[2 \beta_{1 j}^{2} e^{-\beta_{2 j} l} \sin \left(\beta_{1 j} l\right)+\beta_{1 j} \beta_{2 j}-\beta_{1 j} \beta_{2 j} e^{-2 \beta_{2 j} l}\right]\right\} \\
& j=1,2, \cdots,
\end{aligned}
$$

where

$$
\beta_{1 j}=\sqrt{\frac{\sqrt{P^{2}+4 E I \rho \omega_{j}^{2}}-P}{2 E I}}, \quad \beta_{2 j}=\sqrt{\frac{\sqrt{P^{2}+4 E I \rho \omega_{j}^{2}}+P}{2 E I}},
$$

in which $\omega_{j}$ are natural frequencies of the system obtained from the frequency equation

$\operatorname{det}\left[\begin{array}{cc}-I_{m} \omega_{j}^{2}\left[-\beta_{1 j} \sin \left(\beta_{1 j} l\right)-\beta_{2 j} \sinh \left(\beta_{2 j} l\right)\right] & -I_{m} \omega_{j}^{2}\left[\beta_{1 j} \cos \left(\beta_{1 j} l\right)-\beta_{1 j} \cosh \left(\beta_{2 j} l\right)\right] \\ +E I\left[-\beta_{1 j}^{2} \cos \left(\beta_{1 j} l\right)-\beta_{2 j}^{2} \cosh \left(\beta_{2 j} l\right)\right] & +E I\left[-\beta_{1 j}^{2} \sin \left(\beta_{1 j} l\right)-\beta_{1 j} \beta_{2 j} \sinh \left(\beta_{2 j} l\right)\right] \\ \left(k-m \omega_{j}^{2}\right)\left[\cos \left(\beta_{1 j} l\right)-\cosh \left(\beta_{2 j} l\right)\right] & \left(k-m \omega_{j}^{2}\right)\left[\sin \left(\beta_{1 j} l\right)-\frac{\beta_{1 j}}{\beta_{2 j}} \sinh \left(\beta_{2 j} l\right)\right] \\ +P\left[-\beta_{1 j} \sin \left(\beta_{1 j} l\right)-\beta_{2 j} \sinh \left(\beta_{2 j} l\right)\right] & +P\left[\beta_{1 j} \cos \left(\beta_{1 j} l\right)-\beta_{1 j} \cosh \left(\beta_{2 j} l\right)\right] \\ -E I\left[\beta_{1 j}^{3} \sin \left(\beta_{1 j} l\right)-\beta_{2 j}^{3} \sinh \left(\beta_{2 j} l\right)\right] & -E I\left[-\beta_{1 j}^{3} \cos \left(\beta_{1 j} l\right)-\beta_{1 j} \beta_{2 j}^{2} \cosh \left(\beta_{2 j} l\right)\right]\end{array}\right]=0$, 
which can be simplified as

$$
\begin{array}{r}
{\left[2 I_{m} \omega_{j}^{2}\left(k-m \omega_{j}^{2}\right)-E I P\left(\beta_{1 j}^{2}-\beta_{2 j}^{2}\right)-E^{2} I^{2}\left(\beta_{1 j}^{4}+\beta_{2 j}^{4}\right)\right] \beta_{1 j}} \\
+\left[-2 I_{m} \omega_{j}^{2}\left(k-m \omega_{j}^{2}\right)+E I P\left(\beta_{1 j}^{2}-\beta_{2 j}^{2}\right)-2 E^{2} I^{2} \beta_{1 j}^{2} \beta_{2 j}^{2}\right] \beta_{1 j} \cos \left(\beta_{1 j} l\right) \cosh \left(\beta_{2 j} l\right) \\
+\left[I_{m} E I \omega_{j}^{2}\left(\beta_{1 j}^{2}+\beta_{2 j}^{2}\right)+E I\left(k-m \omega_{j}^{2}\right)\left(\frac{\beta_{1 j}^{2}}{\beta_{2 j}^{2}}+1\right)\right] \beta_{1 j} \beta_{2 j} \cos \left(\beta_{1 j} l\right) \sinh \left(\beta_{2 j} l\right) \\
+\left[I_{m} E I \omega_{j}^{2} \beta_{1 j}^{2}-E I\left(k-m \omega_{j}^{2}\right)\right]\left(\beta_{1 j}^{2}+\beta_{2 j}^{2}\right) \sin \left(\beta_{1 j} l\right) \cosh \left(\beta_{2 j} l\right) \\
+\left[I_{m} \omega_{j}^{2}\left(k-m \omega_{j}^{2}\right)\left(1-\frac{\beta_{1 j}^{2}}{\beta_{2 j}^{2}}\right)-2 E I P \beta_{1 j}^{2}-E^{2} I^{2} \beta_{1 j}^{2}\left(\beta_{1 j}^{2}-\beta_{2 j}^{2}\right)\right] \\
\times \beta_{2 j} \sin \left(\beta_{1 j} l\right) \sinh \left(\beta_{2 j} l\right)=0
\end{array}
$$

The exact eigenfunction expansion method uses eigenfunctions of the system as trial functions in Eq. (30), and its results can be used as benchmarks to evaluate performances of the assumed modes method and the new spatial discretization method with two different sets of trial functions chosen for each method. The first set of trial functions, which is used in the assumed modes method, is chosen as the set of eigenfunctions of a clamped-free tensioned beam

$$
\begin{aligned}
\phi_{j}(x)= & \cos \left(\beta_{1 j} x\right)-\frac{2 \beta_{1 j}^{2} e^{-\beta_{2 j} l} \cos \left(\beta_{1 j} l\right)+\beta_{2 j}^{2}+\beta_{2 j}^{2} e^{-2 \beta_{2 j} l}}{2 \beta_{1 j}^{2} e^{-\beta_{2 j} l} \sin \left(\beta_{1 j} l\right)+\beta_{1 j} \beta_{2 j}-\beta_{1 j} \beta_{2 j} e^{-2 \beta_{2 j} l}} \sin \left(\beta_{1 j} x\right) \\
& -\frac{1}{2 \beta_{1 j}^{2} e^{-\beta_{2 j} l} \sin \left(\beta_{1 j} l\right)+\beta_{1 j} \beta_{2 j}-\beta_{1 j} \beta_{2 j} e^{-2 \beta_{2 j} l}} \\
& \times\left(\beta_{1 j}^{2}\left[\sin \left(\beta_{1 j} l\right)-\frac{\beta_{1 j}}{\beta_{2 j}} \cos \left(\beta_{1 j} l\right)-\frac{\beta_{2 j}}{\beta_{1 j}} e^{-\beta_{2 j} l}\right] e^{\beta_{2 j}(x-l)}\right. \\
& \left.+\beta_{1 j}^{2}\left\{e^{-\beta_{2 j} l}\left[\sin \left(\beta_{1 j} l\right)+\frac{\beta_{1 j}}{\beta_{2 j}} \cos \left(\beta_{1 j} l\right)\right]+\frac{\beta_{2 j}}{\beta_{1 j}}\right\} e^{-\beta_{2 j} x}\right), \\
& j=1,2, \cdots, N,
\end{aligned}
$$

where $\omega_{j}$ are obtained from Eq. (89) with $I_{m}=m=k=0$, which satisfy only geometric boundary conditions in Eqs. (67) and (68). The second set of trial functions, which is also used in the assumed modes method, is chosen as

$$
\phi_{j}(x)=\frac{x}{l} \sin \left(\frac{j \pi x}{2 l}\right)+x^{3}, \quad j=1,2, \cdots, N,
$$

which satisfy geometric boundary conditions in Eqs. (67) and (68), and is capable of satisfying natural boundary conditions in Eqs. (69) and (70). In the new spatial discretization method, only two boundary degrees of freedom are required at $x=l$ since the boundary conditions in Eqs. (67) and (68) are homogeneous geometric boundary conditions. With the displacement $e_{3}(t)=u(l, t)$ and the first spatial derivative of the displacement $e_{4}(t)=u_{x}(l, t)$ chosen as boundary degrees of 
freedom, the third set of trial functions, which is used in the new spatial discretization method, is chosen as

$$
\begin{aligned}
\phi_{j}(x)=\varphi_{j}(x)= & \cos \left(\beta_{1 j} x\right)-\frac{2 \beta_{2 j}^{2} e^{-\beta_{2 j} l} \cos \left(\beta_{1 j} l\right)-\beta_{2 j}^{2}-\beta_{2 j}^{2} e^{-2 \beta_{2 j} l}}{2 \beta_{2 j}^{2} e^{-\beta_{2 j} l} \sin \left(\beta_{1 j} l\right)-\beta_{1 j} \beta_{2 j}+\beta_{1 j} \beta_{2 j} e^{-2 \beta_{2 j} l}} \sin \left(\beta_{1 j} x\right) \\
& -\frac{1}{2 \beta_{2 j}^{2} e^{-\beta_{2 j} l} \sin \left(\beta_{1 j} l\right)-\beta_{1 j} \beta_{2 j}+\beta_{1 j} \beta_{2 j} e^{-2 \beta_{2 j} l}} \\
& \times\left(\beta_{2 j}^{2}\left[\sin \left(\beta_{1 j} l\right)-\frac{\beta_{1 j}}{\beta_{2 j}} \cos \left(\beta_{1 j} l\right)+\frac{\beta_{1 j}}{\beta_{2 j}} e^{-\beta_{2 j} l}\right] e^{\beta_{2 j}(x-l)}\right. \\
& \left.+\beta_{2 j}^{2}\left\{e^{-\beta_{2 j} l}\left[\sin \left(\beta_{1 j} l\right)+\frac{\beta_{1 j}}{\beta_{2 j}} \cos \left(\beta_{1 j} l\right)\right]-\frac{\beta_{1 j}}{\beta_{2 j}}\right\} e^{-\beta_{2 j} x}\right), \\
& j=1,2, \cdots, N, \\
\phi_{N+1}(x)=\theta_{3}(x)= & \frac{3 x^{2}}{l^{2}}-\frac{2 x^{3}}{l^{3}}, \\
\phi_{N+2}(x)=\theta_{4}(x)= & \frac{x^{3}}{l^{2}}-\frac{x^{2}}{l},
\end{aligned}
$$

where $\varphi_{j}(x)$ are eigenfunctions of a clamped-clamped tensioned beam, in which $\omega_{j}$ are calculated from the frequency equation of a clamped-clamped tensioned beam

$$
2-2 \cos \left(\beta_{1 j} l\right) \cosh \left(\beta_{2 j} l\right)+\left(\frac{\beta_{2 j}}{\beta_{1 j}}-\frac{\beta_{1 j}}{\beta_{2 j}}\right) \sin \left(\beta_{1 j} l\right) \sinh \left(\beta_{2 j} l\right)=0,
$$

and $\theta_{3}(x)$ and $\theta_{4}(x)$ are polynomials that satisfy Eqs. (21) and (22). With the second spatial derivative of the displacement $e_{3}(t)=u_{x x}(l, t)$ and the third spatial derivative of the displacement $e_{4}(t)=u_{x x x}(l, t)$ chosen as boundary degrees of freedom, the fourth set of trial functions, which is also used in the new spatial discretization method, is chosen as

$$
\begin{aligned}
\phi_{j}(x)=\varphi_{j}(x)= & \cos \left(\beta_{1 j} x\right)-\frac{2 \beta_{1 j}^{2} e^{-\beta_{2 j} l} \cos \left(\beta_{1 j} l\right)+\beta_{2 j}^{2}+\beta_{2 j}^{2} e^{-2 \beta_{2 j} l}}{2 \beta_{1 j}^{2} e^{-\beta_{2 j} l} \sin \left(\beta_{1 j} l\right)+\beta_{1 j} \beta_{2 j}-\beta_{1 j} \beta_{2 j} e^{-2 \beta_{2 j} l}} \sin \left(\beta_{1 j} x\right) \\
& -\frac{1}{2 \beta_{1 j}^{2} e^{-\beta_{2 j} l} \sin \left(\beta_{1 j} l\right)+\beta_{1 j} \beta_{2 j}-\beta_{1 j} \beta_{2 j} e^{-2 \beta_{2 j} l}} \\
& \times\left(\beta_{1 j}^{2}\left[\sin \left(\beta_{1 j} l\right)-\frac{\beta_{1 j}}{\beta_{2 j}} \cos \left(\beta_{1 j} l\right)-\frac{\beta_{2 j}}{\beta_{1 j}} e^{-\beta_{2 j} l}\right] e^{\beta_{2 j}(x-l)}\right. \\
& \left.+\beta_{1 j}^{2}\left\{e^{-\beta_{2 j} l}\left[\sin \left(\beta_{1 j} l\right)+\frac{\beta_{1 j}}{\beta_{2 j}} \cos \left(\beta_{1 j} l\right)\right]+\frac{\beta_{2 j}}{\beta_{1 j}}\right\} e^{-\beta_{2 j} x}\right), \\
& j=1,2, \cdots, N, \\
\phi_{N+1}(x)=\theta_{3}(x)= & \frac{x^{2}}{2}, \\
\phi_{N+2}(x)=\theta_{4}(x)= & \frac{x^{4}}{12 l}-\frac{x^{3}}{6},
\end{aligned}
$$


where $\varphi_{j}(x)$ are eigenfunctions of a clamped-free tensioned beam, in which $\omega_{j}$ are calculated from the frequency equation of a clamped-free tensioned beam

$$
\begin{array}{r}
P\left(\beta_{1 j}^{2}-\beta_{2 j}^{2}\right)+E I\left(\beta_{1 j}^{4}+\beta_{2 j}^{4}\right) \\
+\left[-P\left(\beta_{1 j}^{2}-\beta_{2 j}^{2}\right)+2 E I \beta_{1 j}^{2} \beta_{2 j}^{2}\right] \cos \left(\beta_{1 j} l\right) \cosh \left(\beta_{2 j} l\right) \\
+\left[2 P+E I\left(\beta_{1 j}^{2}-\beta_{2 j}^{2}\right)\right] \beta_{1 j} \beta_{2 j} \sin \left(\beta_{1 j} l\right) \sinh \left(\beta_{2 j} l\right)=0,
\end{array}
$$

and $\theta_{3}(x)$ and $\theta_{4}(x)$ are polynomials that satisfy Eqs. (21) and (22).

\subsection{Natural frequencies and mode shapes}

Parameters of the tensioned beam are given by $l=1, \rho=1, P=1, E I=1, I_{m}=0.01$, $m=1$, and $k=1$. Natural frequencies and mode shapes of the system are calculated by solving the eigenvalue problem

$$
\left(\mathbf{K}^{t b}-\mathbf{M}^{t b} \omega^{2}\right) \boldsymbol{\Phi}=\mathbf{0}
$$

using the four sets of trial functions. Natural frequencies obtained from the four sets of trial functions when $N=10$ are compared with those from the exact eigenvalue problem analysis, and relative errors are shown in Fig. 8. Natural frequencies from the first set of trial functions with the assumed modes method have visible errors for the second through tenth frequencies; those from the second set with the assumed modes method have small errors for the first seven frequencies, but the errors drastically increase for the last three frequencies. Natural frequencies from the third and fourth sets with the new spatial discretization method have overall small errors. The first, second, ninth, and tenth mode shapes calculated from the four sets of trial functions are compared with those from the exact eigenvalue problem analysis, as shown in Fig. 9, and only the third and fourth sets with the new spatial discretization method can give accurate results. 


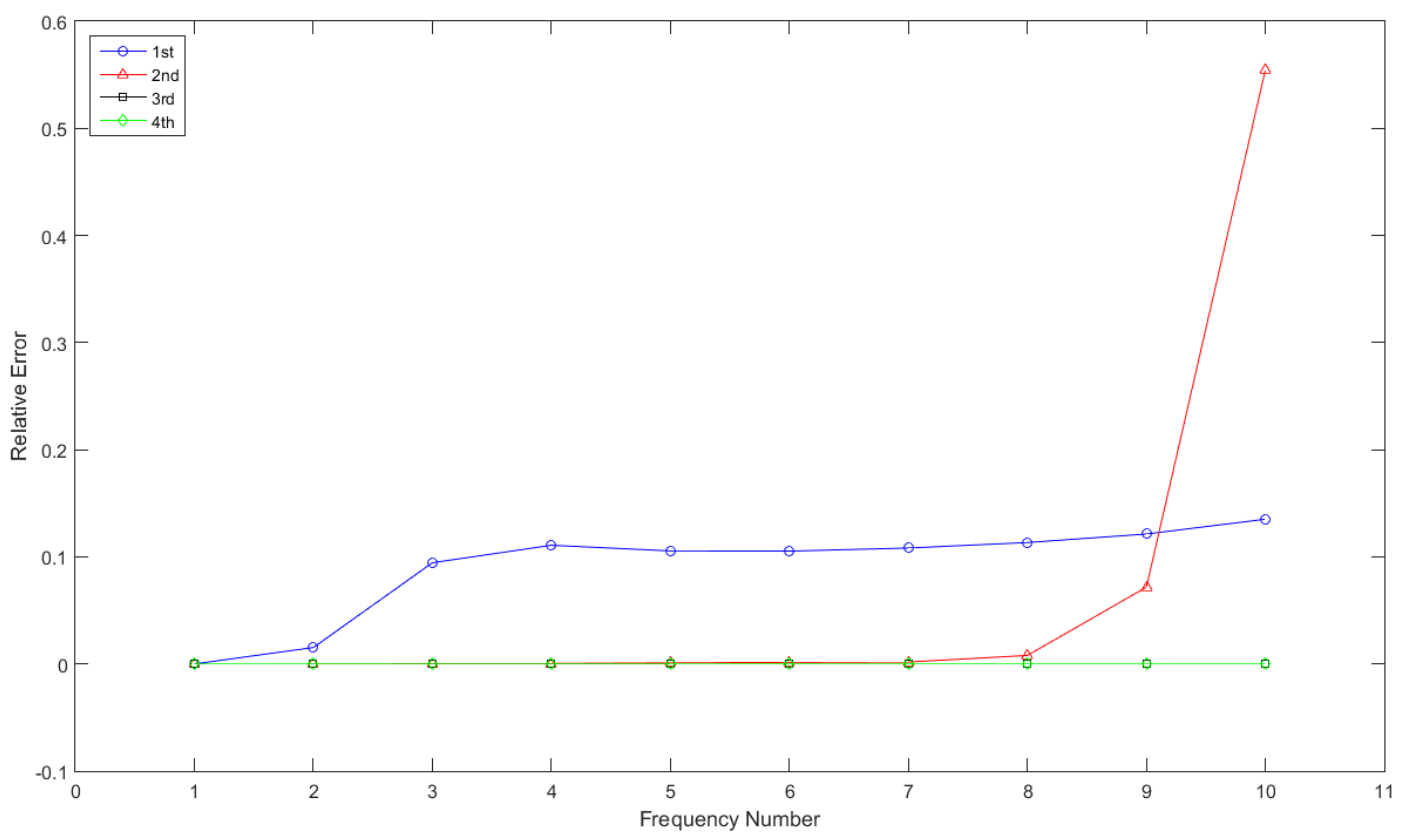

Figure 8: Relative errors of the first 10 natural frequencies of the tensioned beam, where the " $\bigcirc$ ", " $\triangle$ ", " $\square$ ", and " $\diamond$ " signs correspond to the first, second, third, and fourth sets of trial functions, respectively. 

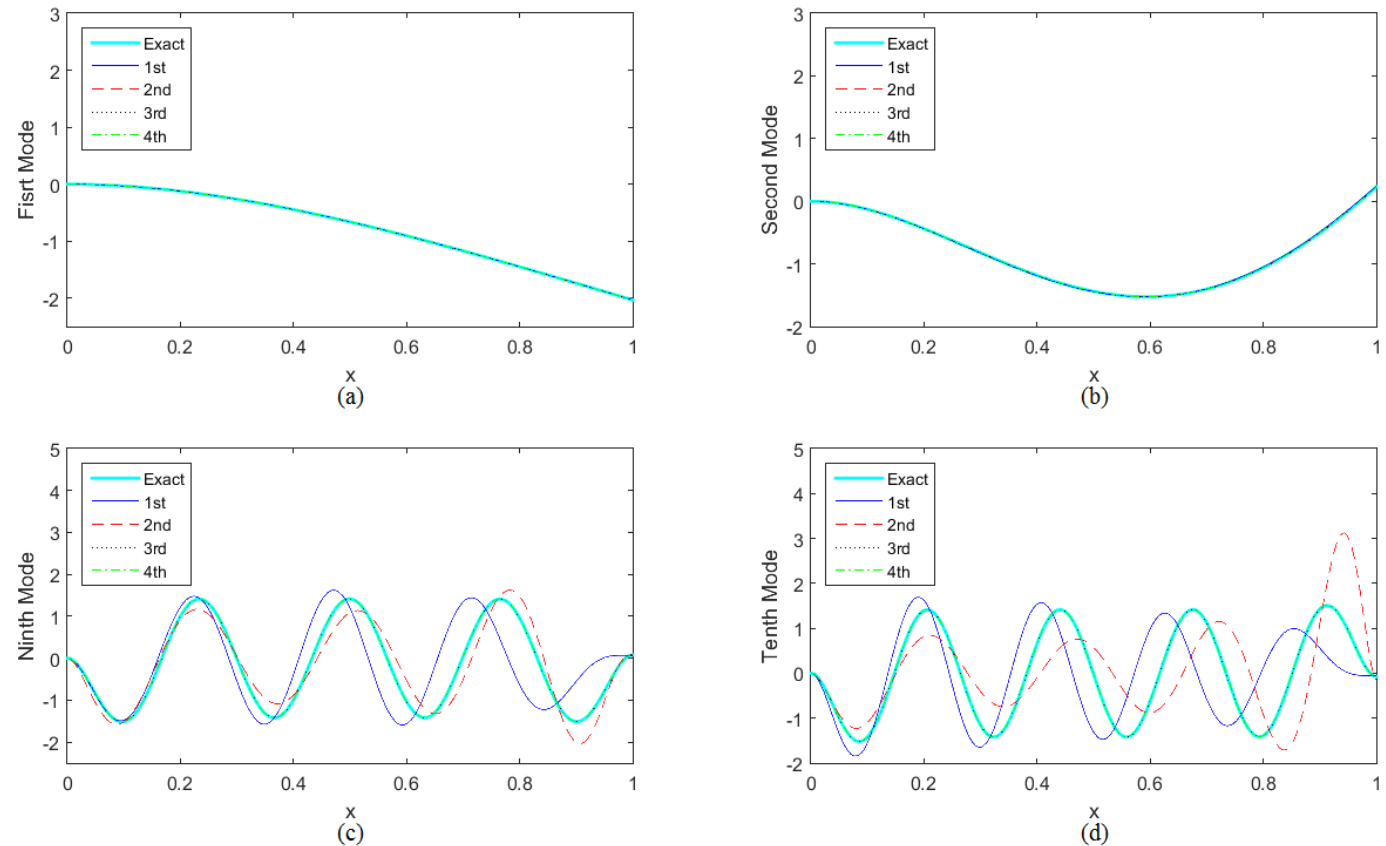

Figure 9: (a) First, (b) second, (c) ninth, and (d) tenth mode shapes of the tensioned beam when $N=10$, where solid, dashed, dotted, and dash-dotted lines correspond to the first, second, third, and fourth sets of trial functions, respectively, and thick solid lines correspond to the exact eigenvalue problem analysis.

\subsection{Harmonic steady-state response}

Parameters of the tensioned beam are the same as those in Sec. 4.1; the external moment and force are $M(t)=M_{0} \sin \left(\omega_{F} t\right)$ and $F(t)=F_{0} \sin \left(\omega_{F} t\right)$, respectively, where $\omega_{F}$ is the excitation frequency. In the exact harmonic steady-state response analysis, the displacement of the beam is assumed to be

$$
u(x, t)=U(x) \sin \left(\omega_{F} t\right) .
$$

Substituting Eq. (101) into Eqs. (66)-(70) yields

$$
\begin{aligned}
U^{\prime \prime \prime \prime}(x)-\frac{P}{E I} U^{\prime \prime}(x)-\frac{\rho}{E I} \omega_{F}^{2} U(x) & =0, \\
U(0) & =0, \\
U^{\prime}(0) & =0, \\
-I_{m} \omega_{F}^{2} U^{\prime}(l)+E I U^{\prime \prime}(l) & =M_{0}, \\
\left(k-m \omega_{F}^{2}\right) U(l)+P U^{\prime}(l)-E I U^{\prime \prime \prime}(l) & =F_{0},
\end{aligned}
$$


whose solution is

$$
U(x)=U_{1}\left[\cos \left(\beta_{1 F} x\right)-\cosh \left(\beta_{2 F} x\right)\right]+U_{2}\left[\sin \left(\beta_{1 F} x\right)-\frac{\beta_{1 F}}{\beta_{2 F}} \sinh \left(\beta_{2 F} x\right)\right]
$$

where

$$
\begin{aligned}
& \beta_{1 F}=\sqrt{\frac{\sqrt{P^{2}+4 E I \rho \omega_{F}^{2}}-P}{2 E I}}, \quad \beta_{2 F}=\sqrt{\frac{\sqrt{P^{2}+4 E I \rho \omega_{F}^{2}}+P}{2 E I}}, \\
& U_{1}=\frac{1}{\triangle}\left(\left\{\left(k-m \omega_{F}^{2}\right)\left[\sin \left(\beta_{1 F} l\right)-\frac{\beta_{1 F}}{\beta_{2 F}} \sinh \left(\beta_{2 F} l\right)\right]\right.\right. \\
& +P\left[\beta_{1 F} \cos \left(\beta_{1 F} l\right)-\beta_{1 F} \cosh \left(\beta_{2 F} l\right)\right] \\
& \left.+E I\left[\beta_{1 F}^{3} \cos \left(\beta_{1 F} l\right)+\beta_{1 F} \beta_{2 F}^{2} \cosh \left(\beta_{2 F} l\right)\right]\right\} M_{0} \\
& -\left\{I_{m} \omega_{F}^{2}\left[-\beta_{1 F} \cos \left(\beta_{1 F} l\right)+\beta_{1 F} \cosh \left(\beta_{2 F} l\right)\right]\right. \\
& \left.\left.-E I\left[\beta_{1 F}^{2} \sin \left(\beta_{1 F} l\right)+\beta_{1 F} \beta_{2 F} \sinh \left(\beta_{2 F} l\right)\right]\right\} F_{0}\right), \\
& U_{2}=\frac{1}{\triangle}\left(-\left\{\left(k-m \omega_{F}^{2}\right)\left[\cos \left(\beta_{1 F} l\right)-\cosh \left(\beta_{2 F} l\right)\right]\right.\right. \\
& -P\left[\beta_{1 F} \sin \left(\beta_{1 F} l\right)+\beta_{2 F} \sinh \left(\beta_{2 F} l\right)\right] \\
& \left.+E I\left[-\beta_{1 F}^{3} \sin \left(\beta_{1 F} l\right)+\beta_{2 F}^{3} \sinh \left(\beta_{2 F} l\right)\right]\right\} M_{0} \\
& +\left\{I_{m} \omega_{F}^{2}\left[\beta_{1 F} \sin \left(\beta_{1 F} l\right)+\beta_{2 F} \sinh \left(\beta_{2 F} l\right)\right]\right. \\
& \left.\left.-E I\left[\beta_{1 F}^{2} \cos \left(\beta_{1 F} l\right)+\beta_{2 F}^{2} \cosh \left(\beta_{2 F} l\right)\right]\right\} F_{0}\right),
\end{aligned}
$$

in which $\triangle$ is the left-hand side of the frequency equation in Eq. (89) with $\beta_{1 j}$ and $\beta_{2 j}$ replaced by $\beta_{1 F}$ and $\beta_{2 F}$, respectively. The displacement of the beam at $x=l$ is given by

$$
\begin{aligned}
u(l, t) & =U(l) \sin \left(\omega_{F} t\right) \\
& =\left\{U_{1}\left[\cos \left(\beta_{1 F} l\right)-\cosh \left(\beta_{2 F} l\right)\right]+U_{2}\left[\sin \left(\beta_{1 F} l\right)-\frac{\beta_{1 F}}{\beta_{2 F}} \sinh \left(\beta_{2 F} l\right)\right]\right\} \sin \left(\omega_{F} t\right)
\end{aligned}
$$

When $\omega_{F} \neq \omega_{j}(j=1,2, \cdots, N)$, its amplitude is

$$
A_{0}=\left|U_{1}\left[\cos \left(\beta_{1 F} l\right)-\cosh \left(\beta_{2 F} l\right)\right]+U_{2}\left[\sin \left(\beta_{1 F} l\right)-\frac{\beta_{1 F}}{\beta_{2 F}} \sinh \left(\beta_{2 F} l\right)\right]\right| .
$$

Note that the amplitude in Eq. (112) approaches infinity when $\omega_{F}$ approaches one of the natural frequencies of the system.

In a trial function expansion method, the generalized coordinate vector has the same form as in Eq. (61). Substituting Eq. (61) into Eq. (82) yields

$$
\left(\mathbf{K}^{t b}-\omega_{F}^{2} \mathbf{M}^{t b}\right) \mathbf{Q}=F_{0} \boldsymbol{\phi}(l)+M_{0} \boldsymbol{\phi}^{\prime}(l),
$$


from which $\mathbf{Q}$ is obtained as

$$
\mathbf{Q}=F_{0}\left(\mathbf{K}^{t b}-\omega_{F}^{2} \mathbf{M}^{t b}\right)^{-1} \boldsymbol{\phi}(l)+M_{0}\left(\mathbf{K}^{t b}-\omega_{F}^{2} \mathbf{M}^{t b}\right)^{-1} \boldsymbol{\phi}^{\prime}(l) .
$$

The displacement of the beam at $x=l$ is given by

$$
\begin{aligned}
u(l, t) & =\boldsymbol{\phi}(l)^{T} \mathbf{Q} \sin \left(\omega_{F} t\right) \\
& =\left[F_{0} \boldsymbol{\phi}(l)^{T}\left(\mathbf{K}^{t b}-\omega_{F}^{2} \mathbf{M}^{t b}\right)^{-1} \boldsymbol{\phi}(l)+M_{0} \boldsymbol{\phi}(l)^{T}\left(\mathbf{K}^{t b}-\omega_{F}^{2} \mathbf{M}^{t b}\right)^{-1} \boldsymbol{\phi}^{\prime}(l)\right] \sin \left(\omega_{F} t\right)(1
\end{aligned}
$$

and its amplitude is

$$
A_{0}=\left|F_{0} \boldsymbol{\phi}(l)^{T}\left(\mathbf{K}^{t b}-\omega_{F}^{2} \mathbf{M}^{t b}\right)^{-1} \boldsymbol{\phi}(l)+M_{0} \boldsymbol{\phi}(l)^{T}\left(\mathbf{K}^{t b}-\omega_{F}^{2} \mathbf{M}^{t b}\right)^{-1} \boldsymbol{\phi}^{\prime}(l)\right|, \quad \omega_{F} \neq \omega_{j},
$$

where $j=1,2, \cdots, N$.

Amplitudes of the displacement $u(l, t)$ with $M_{0}=-1$ and $F_{0}=1$ from the exact harmonic steady-state response analysis and the four sets of trial functions with $N=10$ are shown in Fig. 10. The first and second sets of trial functions with the assumed modes method cannot accurately calculate the amplitudes, and amplitudes calculated from the third and fourth sets of trial functions with the new spatial discretization method match well with that obtained from the exact harmonic steady-state response analysis.

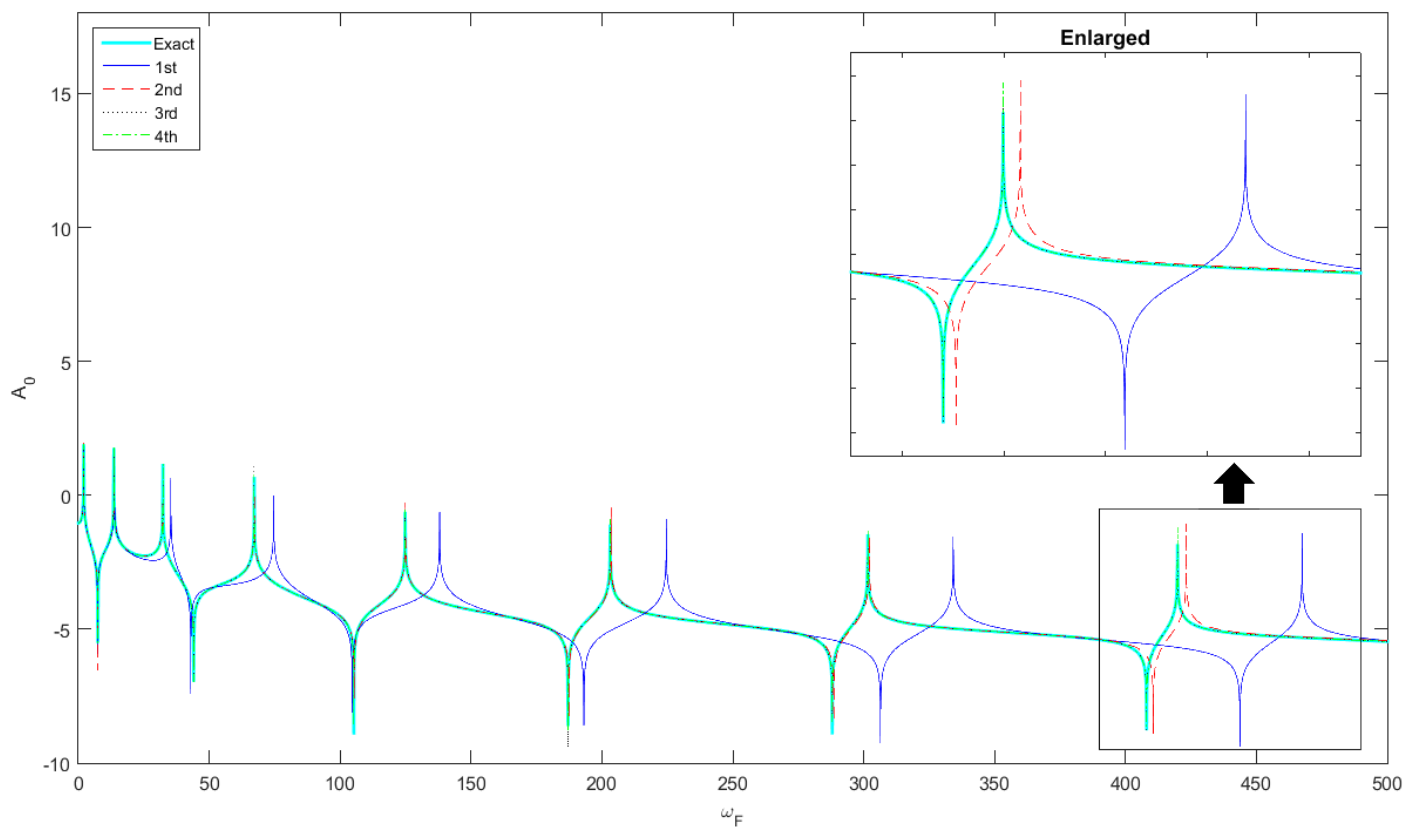

Figure 10: Amplitudes of $u(l, t)$ with varying excitation frequencies, where solid, dashed, dotted, and dash-dotted lines correspond to the first, second, third, and fourth sets of trial functions, respectively, and the thick solid line corresponds to the exact harmonic steady-state response analysis. 


\subsection{Transient response}

Displacements, slopes, bending moments, and shear forces of the system at $t=5$ and at $x=l$ with zero initial conditions and $\omega_{F}=20$ are shown in Figs. 11 and 12, respectively; $N=10$ for the four sets of trial functions. Note that bending moments and shear forces are related to the second and third spatial derivatives of the displacements, respectively. Results from the first and second sets of trial functions are not accurate, and those from the third and fourth sets compare well with those from the exact eigenfunction expansion method and the finite difference method shown in Appendix B with numbers of spatial and temporal nodes being $I=401$ and $J=60001$, respectively.
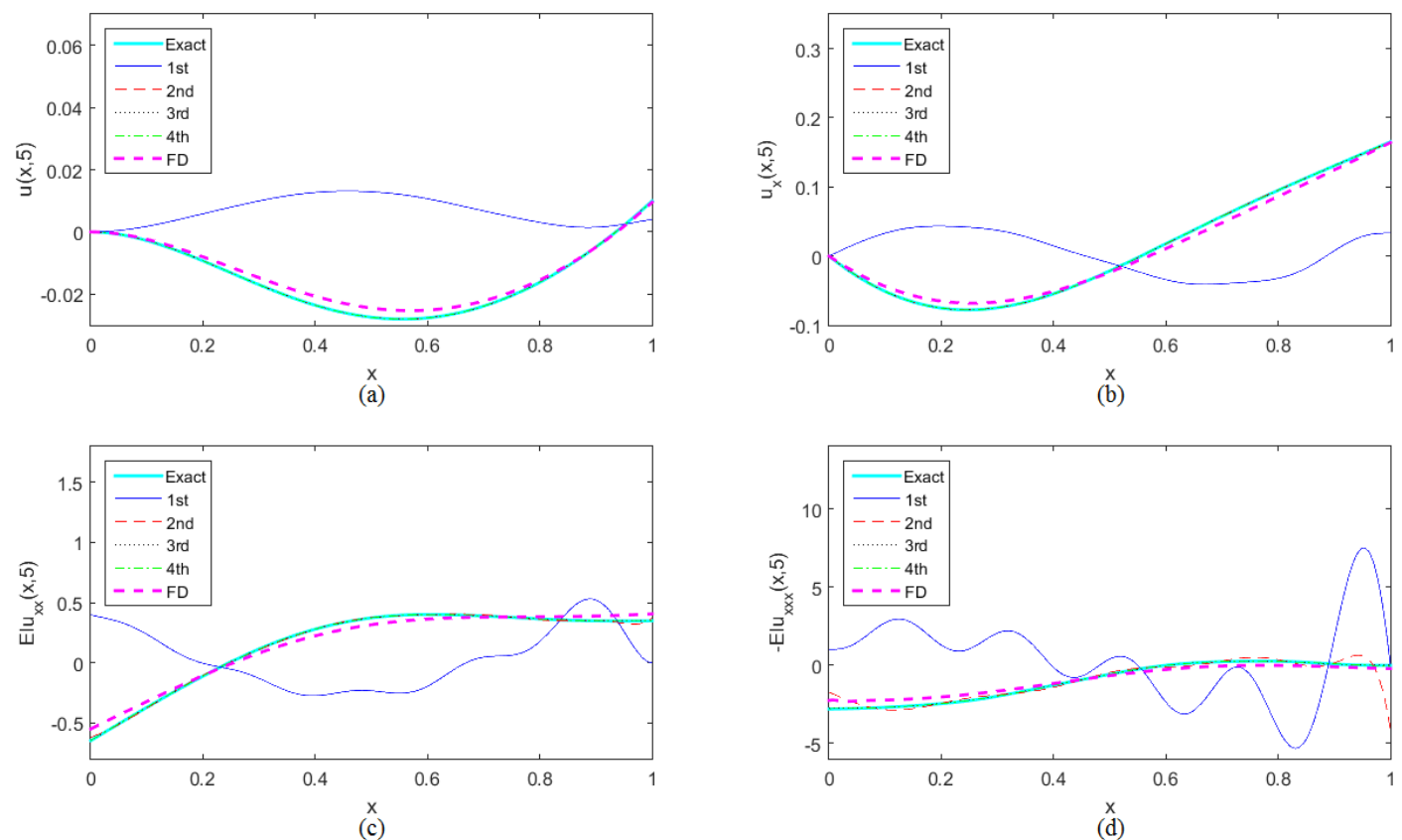

Figure 11: (a) Displacements, (b) slopes, (c) bending moments, and (d) shear forces of the beam at $t=5$, where solid, dashed, dotted, and dash-dotted lines correspond to the first, second, third, and fourth sets of trial functions, respectively, thick solid lines correspond to the exact eigenfunction expansion method, and thick dashed lines correspond to the finite difference (FD) method. 

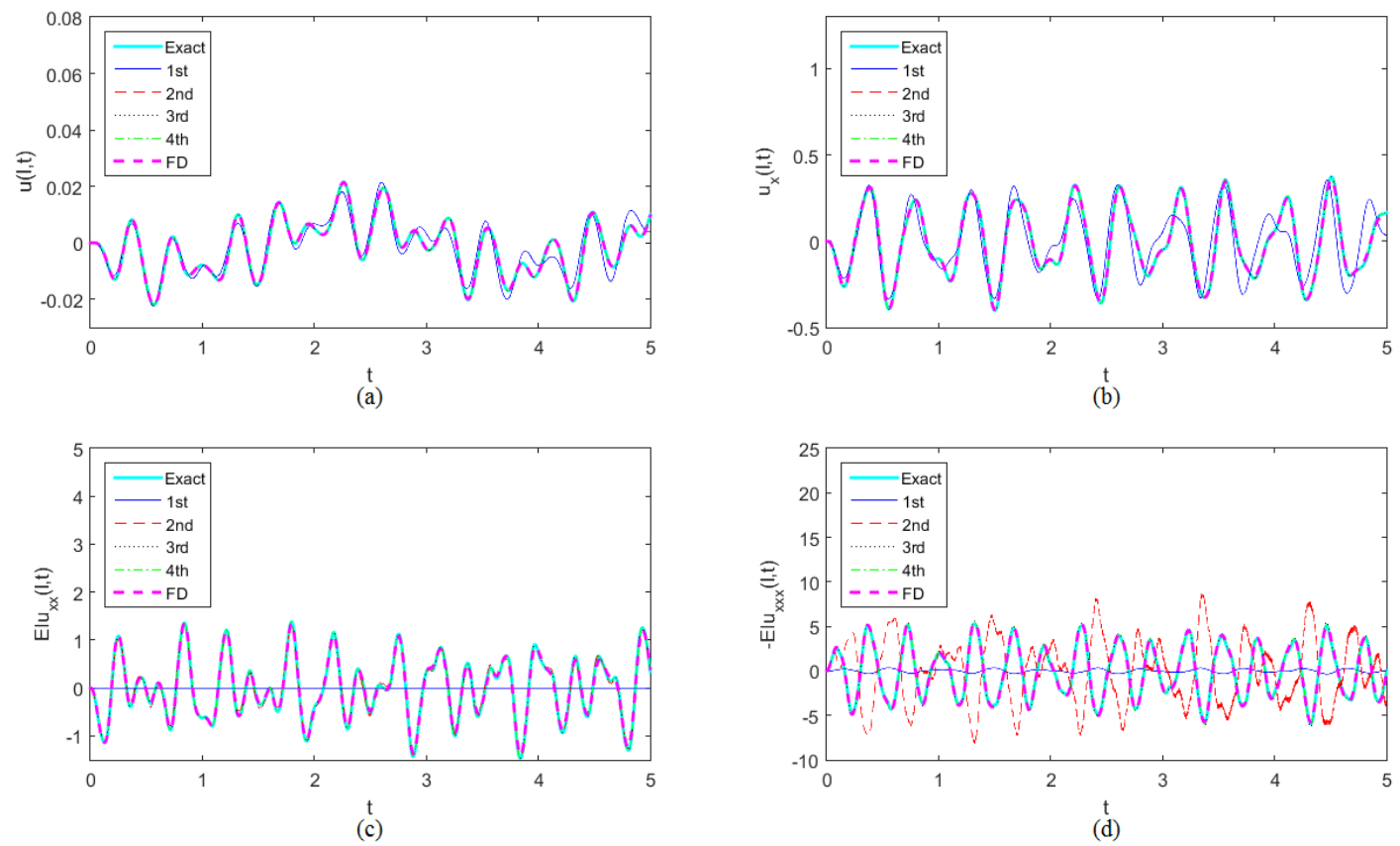

Figure 12: (a) Displacements, (b) slopes, (c) bending moments, and (d) shear forces of the beam at $x=l$, where solid, dashed, dotted, and dash-dotted lines correspond to the first, second, third, and fourth sets of trial functions, respectively, thick solid lines correspond to the exact eigenfunction expansion method, and thick dashed lines correspond to the finite difference (FD) method.

\section{Discussion}

Trial functions for the internal term in the new spatial discretization method are chosen as eigenfunctions of a clamped-clamped or clamped-free tensioned beam in Sec. 4, which satisfy all the geometric boundary conditions of the beam, but involve hyperbolic functions and have rather complicated forms. An alternative choice of trial functions for the internal term are eigenfunctions of a pinned-pinned tensioned beam, which are also eigenfunctions of a pinned-pinned untensioned beam. With $e_{2}(t)=u_{x x}(0, t), e_{3}(t)=u(l, t)$, and $e_{4}(t)=u_{x x}(l, t)$ chosen as boundary degrees of freedom, the fifth set of trial functions of the beam, which is used in the new spatial discretization 
method, is chosen as

$$
\begin{aligned}
\phi_{j}(x)=\varphi_{j}(x) & =\sin \left(j \pi \frac{x}{l}\right), \quad j=1,2, \cdots, N, \\
\phi_{N+1}(x)=\theta_{2}(x) & =-\frac{x^{3}}{6 l}+\frac{x^{2}}{2}-\frac{l x}{3}, \\
\phi_{N+2}(x)=\theta_{3}(x) & =\frac{x}{l}, \\
\phi_{N+3}(x)=\theta_{4}(x) & =\frac{x^{3}}{6 l}-\frac{l x}{6},
\end{aligned}
$$

where $\theta_{2}(x), \theta_{3}(x)$, and $\theta_{4}(x)$ are polynomials that satisfy Eqs. (20)-(22). Note that trial functions of the internal term are sinusoidal functions here, which are much simpler than the eigenfunctions used for the internal terms in the third and fourth sets of trial functions, and they can be used for any fourth-order system. The geometric boundary condition in Eq. (68) is not satisfied by the fifth set of trial functions, and it is used as a constraint to eliminate a generalized coordinate that can be $e_{2}(t), e_{3}(t)$, or $e_{4}(t)$ :

$$
\sum_{j=1}^{N} \phi_{j}^{\prime}(0) p_{j}(t)+\phi_{N+1}^{\prime}(0) e_{2}(t)+\phi_{N+2}^{\prime}(0) e_{3}(t)+\phi_{N+3}^{\prime}(0) e_{4}(t)=0 .
$$

Since $\phi_{N+3}^{\prime}(0)=-l / 6 \neq 0, e_{4}(t)$ can be expressed in terms of $p_{j}(t), e_{2}(t)$, and $e_{3}(t)$ :

$$
e_{4}(t)=-\sum_{j=1}^{N} \frac{\phi_{j}^{\prime}(0)}{\phi_{N+3}^{\prime}(0)} p_{j}(t)-\frac{\phi_{N+1}^{\prime}(0)}{\phi_{N+3}^{\prime}(0)} e_{2}(t)-\frac{\phi_{N+2}^{\prime}(0)}{\phi_{N+3}^{\prime}(0)} e_{3}(t),
$$

and the generalized coordinate vector is converted from a $(N+3) \times 1$ vector to a $(N+2) \times 1$ vector:

$$
\left[\begin{array}{c}
p_{1}(t) \\
\vdots \\
p_{N}(t) \\
e_{2}(t) \\
e_{3}(t) \\
e_{4}(t)
\end{array}\right]=\left[\begin{array}{ccccc}
1 & 0 & \cdots & \cdots & 0 \\
0 & 1 & & & \vdots \\
\vdots & & \ddots & & \vdots \\
\vdots & & & 1 & 0 \\
0 & \cdots & \cdots & 0 & 1 \\
-\frac{\phi_{1}^{\prime}(0)}{\phi_{N+3}^{\prime}(0)} & \cdots & -\frac{\phi_{N}^{\prime}(0)}{\phi_{N+3}^{\prime}(0)} & -\frac{\phi_{N+1}^{\prime}(0)}{\phi_{N+3}^{\prime}(0)} & -\frac{\phi_{N+2}^{\prime}(0)}{\phi_{N+3}^{\prime}(0)}
\end{array}\right]\left[\begin{array}{c}
p_{1}(t) \\
\vdots \\
p_{N}(t) \\
e_{2}(t) \\
e_{3}(t)
\end{array}\right] .
$$

Note that after applying the constraint in Eq. (121), the number of independent generalized coordinates associated with the fifth set of trial functions is $N+2$, which is the same as those associated with the third and fourth sets of trial functions. It is shown in Fig. 13 that squared errors of the displacement of the beam and its first, second, and third spatial derivatives at $t=5$ 
are comparable when calculated from the third, fourth, and fifth sets of trial functions with varying $N$, and the fourth and fifth sets performs better than the third set.

Computational time of the exact eigenfunction expansion method for the beam case is $1.80 \mathrm{~s}$, which is the least time among all the methods. Computational times of the assumed modes method with the first and second sets of trial functions are $2.11 \mathrm{~s}$ and $3.18 \mathrm{~s}$, respectively. However, the natural frequencies, mode shapes, steady-state harmonic responses, and transient responses are not accurately calculated using the assumed modes method. Computational time of the new spatial discretization method with the third through fifth sets of trial functions are $2.94 \mathrm{~s}, 4.53 \mathrm{~s}$, and 3.12 s, respectively. While the new spatial discretization method can take some more computational effort compared with the assumed modes method, all three sets of trial functions in the new spatial discretization method can yield accurate natural frequencies, mode shapes, steady-state harmonic responses, and transient responses of the system. Computational times with the third and fifth sets of trial functions in the new spatial discretization method are less than that of the assumed modes method with the second set of trial functions.
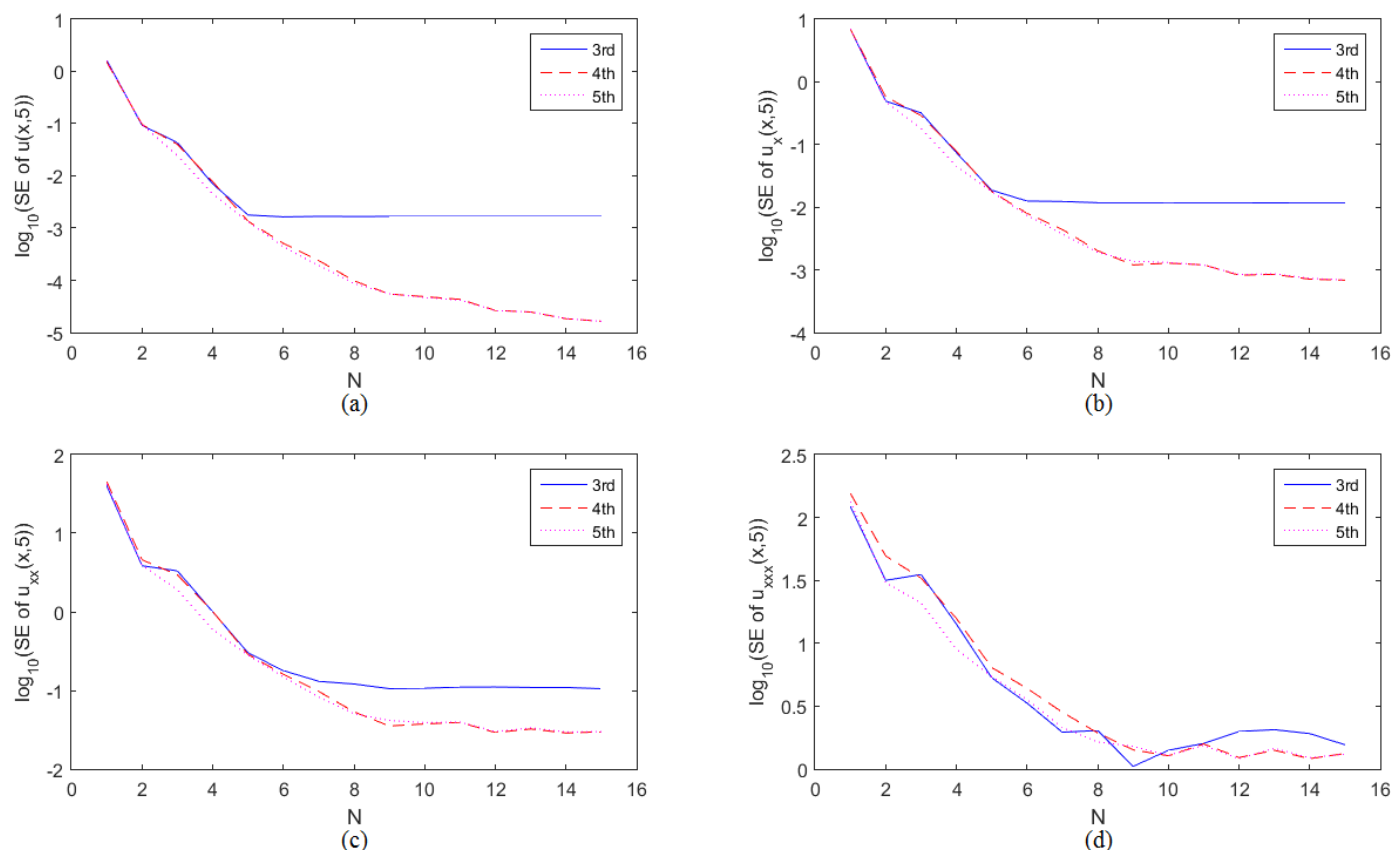

Figure 13: Logarithms of squared errors (SEs) of (a) $u(x, 5),(\mathrm{b}) u_{x}(x, 5)$, (c) $u_{x x}(x, 5)$, and (d) $u_{x x x}(x, 5)$ with varying $N$, where solid, dashed, and dotted lines correspond to the third, fourth, and fifth sets of trial functions, respectively. 
Trial functions of the internal terms in Secs. 3 and 4 are chosen as eigenfunctions of systems with homogeneous boundary conditions, and boundary degrees of freedom and their interpolation functions in the boundary-induced terms remain to be determined. In order to evaluate how the boundary degrees of freedom and their interpolation functions can affect the performance of the new spatial discretization method, squared errors of displacements of the rod and tensioned beam and their first $(K-1)$-th spatial derivatives at $t=5$ are calculated using lower-order boundary degrees of freedom that correspond to zeroth through $(K / 2-1)$-th spatial derivatives of displacements at boundaries and higher-order ones that correspond to $(K / 2)$-th through $(K-1)$-th spatial derivatives of displacements at boundaries, with different interpolation functions. If the lowerorder boundary degree of freedom $u(l, t)$ is chosen for the rod, its first through fourth choices of interpolation functions can be chosen as

$$
\theta_{2}=\frac{x}{l}, \quad \frac{x^{4}}{l^{4}}, \quad \sin \left(\frac{\pi x}{2 l}\right), \text { and } \frac{x}{l} e^{\frac{x}{l}-1},
$$

respectively; if the higher-order boundary degree of freedom $u_{x}(l, t)$ is chosen, its first through fourth choices of interpolation functions can be chosen as

$$
\theta_{2}=x, \quad \frac{x^{4}}{4 l^{3}}, \quad-\frac{l}{\pi} \cos \left(\frac{\pi x}{l}\right), \text { and } \frac{x}{2} e^{\frac{x}{l}-1}
$$

respectively. If lower-order boundary degrees of freedom $u(l, t)$ and $u_{x}(l, t)$ are chosen for the tensioned beam, their first through fourth choices of interpolation functions can be chosen as

$$
\left\{\begin{array}{l}
\theta_{3}=\frac{-2 x^{3}}{l^{3}}+\frac{3 x^{2}}{l^{2}} \\
\theta_{4}=\frac{x^{3}}{l^{2}}-\frac{x^{2}}{l}
\end{array},\left\{\begin{array}{l}
\theta_{3}=\frac{-5 x^{6}}{l^{6}}+\frac{6 x^{5}}{l^{5}} \\
\theta_{4}=\frac{x^{6}}{l^{5}}-\frac{x^{5}}{l^{4}}
\end{array},\left\{\begin{array} { l } 
{ \theta _ { 3 } = \frac { - 8 x ^ { 9 } } { l ^ { 9 } } + \frac { 9 x ^ { 8 } } { l ^ { 8 } } } \\
{ \theta _ { 4 } = \frac { x ^ { 9 } } { l ^ { 8 } } - \frac { x ^ { 8 } } { l ^ { 7 } } }
\end{array} , \text { and } \left\{\begin{array}{l}
\theta_{3}=\frac{-11 x^{12}}{l^{12}}+\frac{12 x^{11}}{l^{11}} \\
\theta_{4}=\frac{x^{12}}{l^{11}}-\frac{x^{11}}{l^{10}}
\end{array},\right.\right.\right.\right.
$$

respectively; if higher-order boundary degrees of freedom $u_{x x}(l, t)$ and $u_{x x x}(l, t)$ are chosen, their first through fourth choices of interpolation functions can be chosen as

$$
\left\{\begin{array}{l}
\theta_{3}=\frac{x^{2}}{2} \\
\theta_{4}=\frac{x^{4}}{12 l}-\frac{x^{3}}{6}
\end{array},\left\{\begin{array}{l}
\theta_{3}=-\frac{x^{6}}{10 l^{4}}+\frac{x^{5}}{5 l^{3}} \\
\theta_{4}=\frac{x^{6}}{30 l^{3}}-\frac{x^{5}}{20 l^{2}}
\end{array},\left\{\begin{array} { l } 
{ \theta _ { 3 } = - \frac { x ^ { 9 } } { 1 2 l ^ { 7 } } + \frac { x ^ { 8 } } { 8 l ^ { 6 } } } \\
{ \theta _ { 4 } = \frac { x ^ { 9 } } { 7 2 l ^ { 6 } } - \frac { x ^ { 8 } } { 5 6 l ^ { 5 } } }
\end{array} , \text { and } \left\{\begin{array}{l}
\theta_{3}=-\frac{3 x^{12}}{44 l^{10}}+\frac{x^{11}}{11 l^{9}} \\
\theta_{4}=\frac{x^{12}}{132 l^{9}}-\frac{x^{11}}{110 l^{8}}
\end{array},\right.\right.\right.\right.
$$

respectively. Results from different interpolation functions are close to each other for the rod and lower-order boundary degrees of freedom work slightly better than higher-order ones, as shown in Fig. 14. However, higher-order boundary degrees of freedom outperform lower-order ones for the tensioned beam and results from different interpolation functions can drastically vary, as shown 
in Fig. 15. It is seen from Figs. 14 and 15 that the new spatial discretization method can ensure convergence of not only displacements of the systems, but also their first $(K-1)$-th spatial derivatives. In general, interpolation functions corresponding to boundary degrees of freedom can be chosen as polynomial, trigonometric, or exponential functions for second-order systems and polynomial functions for fourth-order systems.
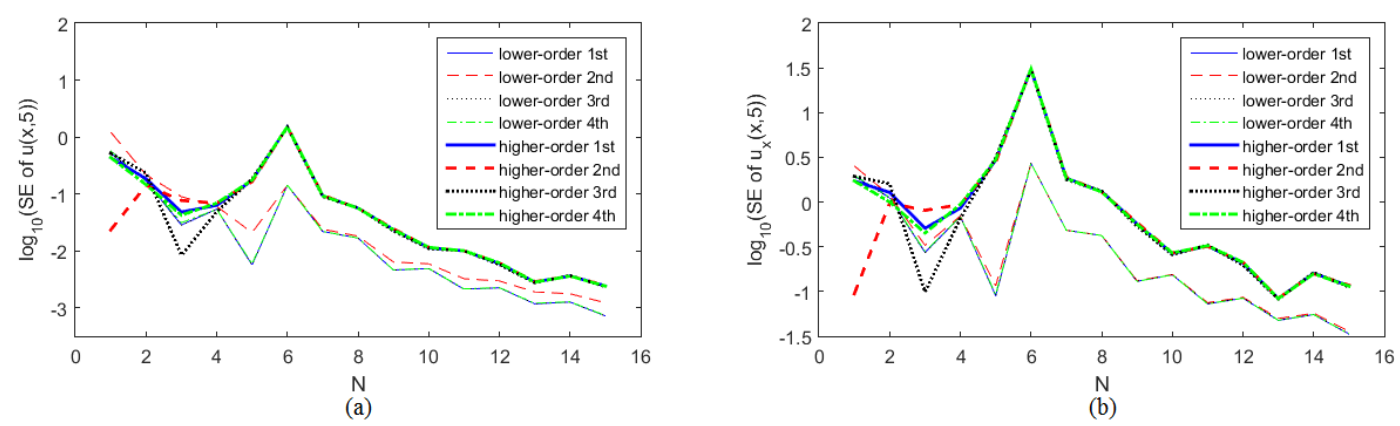

Figure 14: Logarithms of squared errors of (a) $u(x, 5)$ and (b) $u_{x}(x, 5)$ with varying $N$, where thin lines correspond to the lower-order boundary degree of freedom $u(l, t)$, thick lines correspond to the higher-order boundary degree of freedom $u_{x}(l, t)$, and different line types correspond to different interpolation functions for each boundary degree of freedom. 

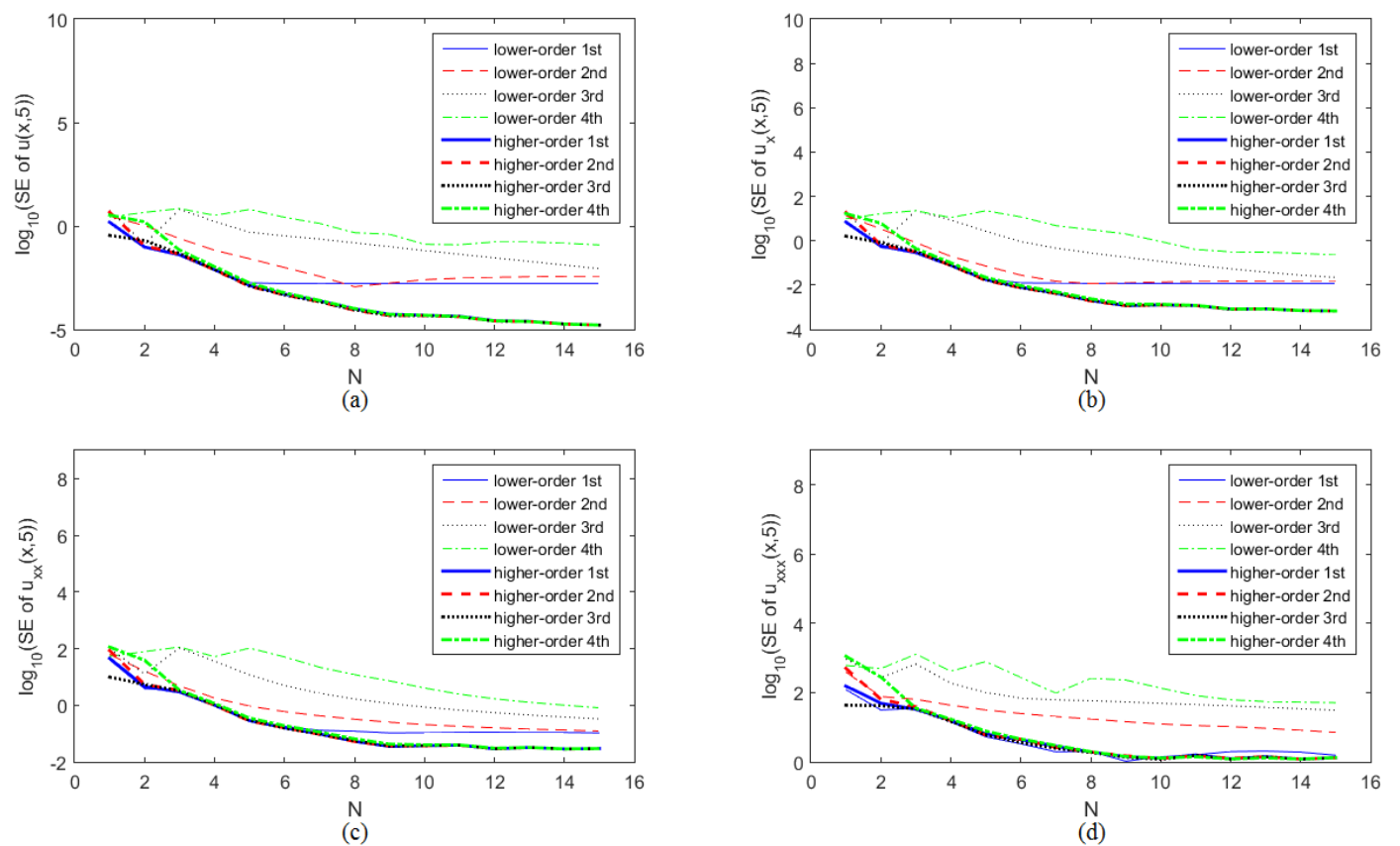

Figure 15: Logarithms of squared errors (SEs) of (a) $u(x, 5)$, (b) $u_{x}(x, 5)$, (c) $u_{x x}(x, 5)$, and (d) $u_{x x x}(x, 5)$ with varying $N$, where thin lines correspond to lower-order boundary degrees of freedom $u(l, t)$ and $u_{x}(l, t)$, thick lines correspond to higher-order boundary degrees of freedom $u_{x x}(l, t)$ and $u_{x x x}(l, t)$, and different line types correspond to different interpolation functions for each choice of boundary degrees of freedom.

An example of the longitudinal vibration of a rod with damping is shown below to demonstrate that the new spatial discretization method can also be used for damped systems. The governing equation of a damped rod is

$$
\rho u_{t t}(x, t)+c u_{t}(x, t)-E A u_{x x}(x, t)=0, \quad 0<x<l, t>0,
$$

where $c$ is the damping coefficient. Since the exact eigenfunction expansion method is not applicable here, the new spatial discretization method is compared with the assumed modes and finite difference methods. Time histories of displacements and axial forces of the damped rod at $x=l$, with zero initial conditions, $c=1$, and $\omega_{F}=20$, are shown in Fig. 16, which shows that the new spatial discretization method can accurately calculate the displacement and axial force of the rod with the displacement decreasing with time due to damping. Similar to the rod case without damping, the assumed modes method using the first set of trial functions cannot accurately calculate the axial force. 

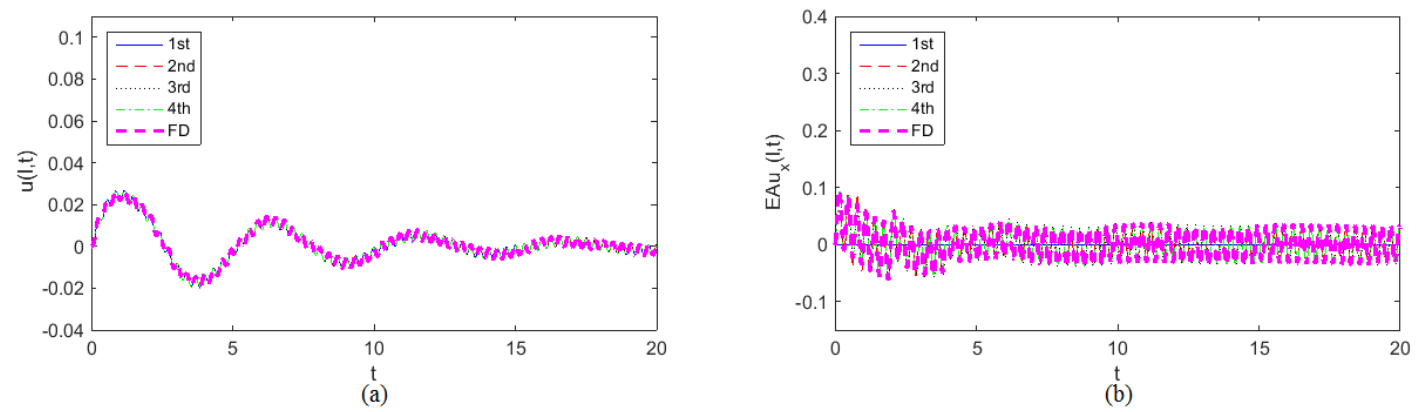

Figure 16: (a) Displacements and (b) axial forces of the damped rod at $x=l$ with $c=1$ and $\omega_{F}=20$, where solid, dashed, dotted, and dash-dotted lines correspond to the first, second, third, and fourth sets of trial functions, respectively, and thick dashed lines correspond to the finite difference (FD) method.

\section{Conclusion}

The new spatial discretization method is applicable to a one-dimensional continuous system with arbitrary boundary conditions, and it spatially discretizes the displacement of the system into an internal and a boundary-induced term, where the internal term satisfies certain prescribed homogeneous boundary conditions and the boundary-induced term uses additional boundary degrees of freedom to account for actual boundary conditions. A general formulation of the new spatial discretization method is provided for both second- and fourth-order continuous systems. Natural frequencies, mode shapes, harmonic steady-state responses, and transient responses of a rod and tensioned beam with mass-spring sub-systems attached to their boundaries are calculated using the new spatial discretization method and the assumed modes method. It is shown that results from the assumed modes method are not accurate, and those from the new spatial discretization method compare well with those from exact analyses and the finite difference method for transient responses. Unlike the exact eigenfunction expansion method that has orthogonal trial functions, the new spatial discretization method has most of its trial functions orthogonal and up to $K$ ones that are not. Unlike the assumed modes method with quasi-comparison functions used as trial functions, whose mass matrix has a fast growing condition number with the increase of $N$, that in the new spatial discretization method grows much more slowly, which makes it feasible to deal with problems that require a large number of truncated terms to achieve convergence. While the exact eigenfunction expansion method usually uses complicated eigenfunctions as trial functions 
for fourth-order systems, sinusoidal functions can be used as trial functions of the internal term for any fourth-order system in the new spatial discretization method, and geometric boundary conditions of the system that are not satisfied by the trial functions need to be imposed as constraints to accurately solve for the displacement of the system and its first $(K-1)$-th spatial derivatives. Lower-order boundary degrees of freedom work slightly better than higher-order ones for the rod, and higher-order ones can achieve better convergence than lower-order ones for the tensioned beam. While the new spatial discretization method uses some additional boundary degrees of freedom whose trial functions are not orthogonal to those of the internal term, its computational effort can still be less than that of the assumed modes method if the trial functions are properly chosen.

\section{Acknowledgment}

The authors are grateful for the financial support from the National Science Foundation through Award CMMI-1000830 and the National Science Foundation of China through Award No. 11442006.

\section{Appendix A. Finite Difference Algorithm for the Rod}

Spatial and temporal domains are evenly discretized into a grid with the spatial step $\xi=$ $l /(I-1)$ and the temporal step $\eta=5 /(J-1)$, so that

$$
\begin{aligned}
x_{i} & =(i-1) \xi, \quad i=1,2, \cdots, I \\
t_{j} & =(j-1) \eta, \quad j=1,2, \cdots, J
\end{aligned}
$$

are the $i$-th spatial point and the $j$-th temporal point, respectively. The displacement $u(x, t)$ at the grid point $\left(x_{i}, t_{j}\right)$ is defined by

$$
u_{i}^{j}=u\left(x_{i}, t_{j}\right) .
$$

The forward time central space scheme [10] is implemented for $u\left(x_{i}, t_{j}\right)$; some partial derivatives of $u$ with respect to $t$ and $x$ are 


$$
\begin{aligned}
\frac{\partial^{2} u\left(x_{i}, t_{j}\right)}{\partial t^{2}} & =\frac{v_{i}^{j}-v_{i}^{j-1}}{\eta}, \\
\frac{\partial u\left(x_{i}, t_{j}\right)}{\partial x} & =\frac{u_{i+1}^{j}-u_{i-1}^{j}}{2 \xi}, \\
\frac{\partial^{2} u\left(x_{i}, t_{j}\right)}{\partial x^{2}} & =\frac{u_{i+1}^{j}-2 u_{i}^{j}+u_{i-1}^{j}}{\xi^{2}},
\end{aligned}
$$

where $v_{i}^{j}=\left(u_{i}^{j}-u_{i}^{j-1}\right) / \eta$ is the velocity at the grid point $\left(x_{i}, t_{j}\right)$. Substituting Eqs. (A.3)-(A.6) into Eqs. (27)-(29) yield

$$
\begin{aligned}
\rho \frac{v_{i}^{j}-v_{i}^{j-1}}{\eta}-E A \frac{u_{i+1}^{j}-2 u_{i}^{j}+u_{i-1}^{j}}{\xi^{2}} & =0, i=2, \cdots, I-1, \\
u_{1}^{j} & =0, \\
m \frac{v_{I}^{j}-v_{I}^{j-1}}{\eta}+k u_{I}^{j}+E A \frac{u_{I+1}^{j}-u_{I-1}^{j}}{2 \xi} & =F_{j},
\end{aligned}
$$

where $j=2, \cdots, J$ and $F_{j}=F\left(t_{j}\right)$. Rearranging Eq. (A.7) yields

$$
-\frac{E A}{\xi^{2}} u_{i-1}^{j}+\left(\frac{\rho}{\eta^{2}}+\frac{2 E A}{\xi^{2}}\right) u_{i}^{j}-\frac{E A}{\xi^{2}} u_{i+1}^{j}=\frac{\rho}{\eta} v_{i}^{j-1}+\frac{\rho}{\eta^{2}} u_{i}^{j-1}, i=2, \cdots, I-1 .
$$

By setting $i=1$ and $i=I$ in Eq. (A.10), $u_{0}^{j}$ and $u_{I+1}^{j}$ can be written as

$$
\begin{aligned}
u_{0}^{j} & =-\frac{\rho \xi^{2}}{E A \eta} v_{1}^{j-1}-\frac{\rho \xi^{2}}{E A \eta^{2}} u_{1}^{j-1}+\left(\frac{\rho \xi^{2}}{E A \eta^{2}}+2\right) u_{1}^{j}-u_{2}^{j}, \\
u_{I+1}^{j} & =-\frac{\rho \xi^{2}}{E A \eta} v_{I}^{j-1}-\frac{\rho \xi^{2}}{E A \eta^{2}} u_{I}^{j-1}-u_{I-1}^{j}+\left(\frac{\rho \xi^{2}}{E A \eta^{2}}+2\right) u_{I}^{j} .
\end{aligned}
$$

Substituting Eq. (A.12) into Eq. (A.9) yields

$$
-\frac{E A}{\xi} u_{I-1}^{j}+\left(\frac{\rho \xi}{2 \eta^{2}}+\frac{E A}{\xi}+\frac{m}{\eta^{2}}+k\right) u_{I}^{j}=F_{j}+\left(\frac{\rho \xi}{2 \eta}+\frac{m}{\eta}\right) v_{I}^{j-1}+\left(\frac{\rho \xi}{2 \eta^{2}}+\frac{m}{\eta^{2}}\right) u_{I}^{j-1} .
$$

By combining Eqs. (A.8), (A.10), and (A.13), a system of $I$ linear algebraic equations are constructed to obtain displacements at the time level $j(j=2, \cdots, J)$ from displacements and velocities at the time level $j-1$ :

$$
\mathbf{X}^{r} \mathbf{u}^{j}=\mathbf{Y}^{r}
$$


where $\mathbf{u}^{j}=\left[\begin{array}{llll}u_{1}^{j} & u_{2}^{j} & \cdots & u_{I}^{j}\end{array}\right]^{T}$ is the displacement vector at the time level $j$,

$$
\begin{aligned}
& \mathbf{X}^{r}=\left[\begin{array}{cccccc}
1 & 0 & \cdots & \cdots & \cdots & 0 \\
\left(-\frac{E A}{\xi^{2}}\right) & \left(\frac{\rho}{\eta^{2}}+\frac{2 E A}{\xi^{2}}\right) & \left(-\frac{E A}{\xi^{2}}\right) & 0 & \cdots & 0 \\
0 & \ddots & \ddots & \ddots & \ddots & \vdots \\
\vdots & \ddots & \ddots & \ddots & \ddots & 0 \\
0 & \cdots & 0 & \left(-\frac{E A}{\xi^{2}}\right) & \left(\frac{\rho}{\eta^{2}}+\frac{2 E A}{\xi^{2}}\right) & \left(-\frac{E A}{\xi^{2}}\right) \\
0 & \cdots & \cdots & 0 & \left(-\frac{E A}{\xi}\right) & \left(\frac{\rho \xi}{2 \eta^{2}}+\frac{E A}{\xi}+\frac{m}{\eta^{2}}+k\right)
\end{array}\right] \\
& \mathbf{Y}^{r}=\left[\begin{array}{c}
0 \\
\frac{\rho}{\eta} v_{2}^{j-1}+\frac{\rho}{\eta^{2}} u_{2}^{j-1} \\
\vdots \\
\vdots \\
\frac{\rho}{\eta} v_{I-1}^{j-1}+\frac{\rho}{\eta^{2}} u_{I-1}^{j-1} \\
F_{j}+\left(\frac{\rho \xi}{2 \eta}+\frac{m}{\eta}\right) v_{I}^{j-1}+\left(\frac{\rho \xi}{2 \eta^{2}}+\frac{m}{\eta^{2}}\right) u_{I}^{j-1}
\end{array}\right]
\end{aligned}
$$

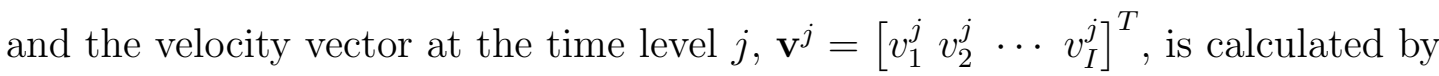

$$
\mathbf{v}^{j}=\frac{\mathbf{u}^{j}-\mathbf{u}^{j-1}}{\eta} .
$$

Displacement and velocity vectors at the first time level are determined from initial conditions of the system. After obtaining displacements at the time level $j, u_{0}^{j}$ and $u_{I+1}^{j}$ can be calculated from Eqs. (A.11) and (A.12), respectively, and the first spatial derivatives of the displacements are calculated from Eq. (A.5) with $i=1, \cdots, I$.

\section{Appendix B. Finite Difference Algorithm for the Tensioned Beam}

The same $I \times J$ grid as in Appendix A is used here. In addition to Eqs. (A.4)-(A.6), some partial derivatives of $u$ with respect $x$ are

$$
\begin{aligned}
\frac{\partial^{3} u\left(x_{i}, t_{j}\right)}{\partial x \partial t^{2}} & =\frac{v_{i+1}^{j}-v_{i-1}^{j}-v_{i+1}^{j-1}+v_{i-1}^{j-1}}{2 \xi \eta}, \\
\frac{\partial^{3} u\left(x_{i}, t_{j}\right)}{\partial x^{3}} & =\frac{u_{i+2}^{j}-2 u_{i+1}^{j}+2 u_{i-1}^{j}-u_{i-2}^{j}}{2 \xi^{3}}, \\
\frac{\partial^{4} u\left(x_{i}, t_{j}\right)}{\partial x^{4}} & =\frac{u_{i+2}^{j}-4 u_{i+1}^{j}+6 u_{i}^{j}-4 u_{i-1}^{j}+u_{i-2}^{j}}{\xi^{4}} .
\end{aligned}
$$


Substituting Eqs. (A.3), (A.4), (A.5), (A.6), (B.1), (B.2), and (B.3) into Eqs. (66)-(70) yield

$$
\begin{aligned}
\rho \frac{v_{i}^{j}-v_{i}^{j-1}}{\eta}-P \frac{u_{i+1}^{j}-2 u_{i}^{j}+u_{i-1}^{j}}{\xi^{2}} & \\
+E I \frac{u_{i+2}^{j}-4 u_{i+1}^{j}+6 u_{i}^{j}-4 u_{i-1}^{j}+u_{i-2}^{j}}{\xi^{4}} & =0, i=3, \cdots, I-2, \\
u_{1}^{j} & =0, \\
\frac{u_{2}^{j}-u_{0}^{j}}{2 \xi} & =0, \\
I_{m} \frac{v_{I+1}^{j}-v_{I-1}^{j}-v_{I+1}^{j-1}+v_{I-1}^{j-1}}{2 \xi \eta} & =M_{j}, \\
m \frac{v_{I}^{j}-v_{I}^{j-1}}{\eta}+k u_{I}^{j}+P \frac{u_{I+1}^{j}-2 u_{I}^{j}+u_{I-1}^{j}}{\xi^{2}} & \\
-E I \frac{u_{I+2}^{j}-2 u_{I-1}^{j}}{2 u_{I+1}^{j}+2 u_{I-1}^{j}-u_{I-2}^{j}} & =F_{j},
\end{aligned}
$$

where $j=2, \cdots, J$ and $M_{j}=M\left(t_{j}\right)$. Rearranging Eq. (B.4) yields

$$
\begin{aligned}
\frac{E I}{\xi^{4}} u_{i-2}^{j}-\left(\frac{P}{\xi^{2}}+\frac{4 E I}{\xi^{4}}\right) & u_{i-1}^{j}+\left(\frac{\rho}{\eta^{2}}+\frac{2 P}{\xi^{2}}+\frac{6 E I}{\xi^{4}}\right) u_{i}^{j} \\
-\left(\frac{P}{\xi^{2}}+\frac{4 E I}{\xi^{4}}\right) u_{i+1}^{j}+\frac{E I}{\xi^{4}} u_{i+2}^{j}= & \frac{\rho}{\eta} v_{i}^{j-1}+\frac{\rho}{\eta^{2}} u_{i}^{j-1}, \\
& i=3, \cdots, I-2,
\end{aligned}
$$

By setting $i=2$ and $i=I-1$ in Eq. (B.9), $u_{0}^{j}$ and $u_{I+1}^{j}$ can be written as

$$
\begin{aligned}
u_{0}^{j}= & \frac{\rho \xi^{4}}{E I \eta} v_{2}^{j-1}+\frac{\rho \xi^{4}}{E I \eta^{2}} u_{2}^{j-1} \\
& +\left(\frac{P \xi^{2}}{E I}+4\right) u_{1}^{j}-\left(\frac{\rho \xi^{4}}{E I \eta^{2}}+\frac{2 P \xi^{2}}{E I}+6\right) u_{2}^{j}+\left(\frac{P \xi^{2}}{E I}+4\right) u_{3}^{j}-u_{4}^{j}, \\
u_{I+1}^{j}= & \frac{\rho \xi^{4}}{E I \eta} v_{I-1}^{j-1}+\frac{\rho \xi^{4}}{E I \eta^{2}} u_{I-1}^{j-1} \\
& -u_{I-3}^{j}+\left(\frac{P \xi^{2}}{E I}+4\right) u_{I-2}^{j}-\left(\frac{\rho \xi^{4}}{E I \eta^{2}}+\frac{2 P \xi^{2}}{E I}+6\right) u_{I-1}^{j}+\left(\frac{P \xi^{2}}{E I}+4\right) u_{I}^{j} .
\end{aligned}
$$


By setting $i=1$ and $i=I$ in Eq. (B.9) and using Eqs. (B.10) and (B.11), $u_{-1}^{j}$ and $u_{I+2}^{j}$ can be written as

$$
\begin{aligned}
u_{-1}^{j}= & \frac{\rho \xi^{4}}{E I \eta} v_{1}^{j-1}+\frac{\rho \xi^{4}}{E I \eta^{2}} u_{1}^{j-1}+\left(\frac{\rho P \xi^{6}}{E^{2} I^{2} \eta}+\frac{4 \rho \xi^{4}}{E I \eta}\right) v_{2}^{j-1}+\left(\frac{\rho P \xi^{6}}{E^{2} I^{2} \eta^{2}}+\frac{4 \rho \xi^{4}}{E I \eta^{2}}\right) u_{2}^{j-1} \\
& +\left(\frac{P^{2} \xi^{4}}{E^{2} I^{2}}-\frac{\rho \xi^{4}}{E I \eta^{2}}+\frac{6 P \xi^{2}}{E I}+10\right) u_{1}^{j}-\left(\frac{\rho P \xi^{6}}{E^{2} I^{2} \eta^{2}}+\frac{2 P^{2} \xi^{4}}{E^{2} I^{2}}+\frac{4 \rho \xi^{4}}{E I \eta^{2}}+\frac{13 P \xi^{2}}{E I}+20\right) u_{2}^{j} \\
& +\left(\frac{P^{2} \xi^{4}}{E^{2} I^{2}}+\frac{8 P \xi^{2}}{E I}+15\right) u_{3}^{j}-\left(\frac{P \xi^{2}}{E I}+4\right) u_{4}^{j}, \\
u_{I+2}^{j}= & \left(\frac{\rho P \xi^{6}}{E^{2} I^{2} \eta}+\frac{4 \rho \xi^{4}}{E I \eta}\right) v_{I-1}^{j-1}+\left(\frac{\rho P \xi^{6}}{E^{2} I^{2} \eta^{2}}+\frac{4 \rho \xi^{4}}{E I \eta^{2}}\right) u_{I-1}^{j-1}+\frac{\rho \xi^{4}}{E I \eta} v_{I}^{j-1}+\frac{\rho \xi^{4}}{E I \eta^{2}} u_{I}^{j-1} \\
& -\left(\frac{P \xi^{2}}{E I}+4\right) u_{I-3}^{j}+\left(\frac{P^{2} \xi^{4}}{E^{2} I^{2}}+\frac{8 P \xi^{2}}{E I}+15\right) u_{I-2}^{j} \\
& -\left(\frac{\rho P \xi^{6}}{E^{2} I^{2} \eta^{2}}+\frac{2 P^{2} \xi^{4}}{E^{2} I^{2}}+\frac{4 \rho \xi^{4}}{E I \eta^{2}}+\frac{13 P \xi^{2}}{E I}+20\right) u_{I-1}^{j} \\
& +\left(\frac{P^{2} \xi^{4}}{E^{2} I^{2}}-\frac{\rho \xi^{4}}{E I \eta^{2}}+\frac{6 P \xi^{2}}{E I}+10\right) u_{I}^{j} .
\end{aligned}
$$


Substituting Eq. (B.10) into Eq. (B.6), Eq. (B.11) into Eq. (B.7), and Eqs. (B.11) and (B.13) into Eq. (B.8) yield

$$
\begin{aligned}
& -\left(\frac{P}{\xi^{2}}+\frac{4 E I}{\xi^{4}}\right) u_{1}^{j}+\left(\frac{\rho}{\eta^{2}}+\frac{2 P}{\xi^{2}}+\frac{7 E I}{\xi^{4}}\right) u_{2}^{j} \\
& -\left(\frac{P}{\xi^{2}}+\frac{4 E I}{\xi^{4}}\right) u_{3}^{j}+\frac{E I}{\xi^{4}} u_{4}^{j}=\frac{\rho}{\eta} v_{2}^{j-1}+\frac{\rho}{\eta^{2}} u_{2}^{j-1}, \\
& -\left(\frac{E I}{\xi^{2}}+\frac{I_{m}}{2 \xi \eta^{2}}\right) u_{I-3}^{j}+\left(P+\frac{4 E I}{\xi^{2}}+\frac{2 I_{m}}{\xi \eta^{2}}+\frac{P I_{m} \xi}{2 E I \eta^{2}}\right) u_{I-2}^{j} \\
& -\left(\frac{\rho \xi^{2}}{\eta^{2}}+2 P+\frac{5 E I}{\xi^{2}}+\frac{7 I_{m}}{2 \xi \eta^{2}}+\frac{\rho I_{m} \xi^{3}}{2 E I \eta^{4}}+\frac{P I_{m} \xi}{E I \eta^{2}}\right) u_{I-1}^{j} \\
& +\left(P+\frac{2 E I}{\xi^{2}}+\frac{2 I_{m}}{\xi \eta^{2}}+\frac{P I_{m} \xi}{2 E I \eta^{2}}\right) u_{I}^{j}=M_{j} \\
& -\left(\frac{\rho \xi^{2}}{\eta}+\frac{I_{m}}{2 \xi \eta}+\frac{\rho I_{m} \xi^{3}}{2 E I \eta^{3}}\right) v_{I-1}^{j-1} \\
& -\left(\frac{\rho \xi^{2}}{\eta^{2}}+\frac{I_{m}}{2 \xi \eta^{2}}+\frac{\rho I_{m} \xi^{3}}{2 E I \eta^{4}}\right) u_{I-1}^{j-1} \\
& +\frac{I_{m}}{2 \xi \eta} v_{I+1}^{j-1}+\frac{I_{m}}{2 \xi \eta^{2}} u_{I+1}^{j-1} \\
& \frac{E I}{\xi^{3}} u_{I-3}^{j}-\left(\frac{P}{\xi}+\frac{3 E I}{\xi^{3}}\right) u_{I-2}^{j}+\left(\frac{\rho \xi}{\eta^{2}}+\frac{P}{\xi}+\frac{3 E I}{\xi^{3}}\right) u_{I-1}^{j} \\
& +\left(\frac{\rho \xi}{2 \eta^{2}}-\frac{E I}{\xi^{3}}+\frac{m}{\eta^{2}}+k\right) u_{I}^{j}=F_{j}+\frac{\rho \xi}{\eta} v_{I-1}^{j-1}+\frac{\rho \xi}{\eta^{2}} u_{I-1}^{j-1} \\
& +\left(\frac{\rho \xi}{2 \eta}+\frac{m}{\eta}\right) v_{I}^{j-1} \\
& +\left(\frac{\rho \xi}{2 \eta^{2}}+\frac{m}{\eta^{2}}\right) u_{I}^{j-1} \text {. }
\end{aligned}
$$

By combining Eqs. (B.5), (B.14), (B.9), (B.15), and (B.16), a system of $I$ linear algebraic equations are constructed to obtain displacements at the time level $j(j=2, \cdots, J)$ from displacements and velocities at the time level $j-1$ :

$$
\mathbf{X}^{t b} \mathbf{u}^{j}=\mathbf{Y}^{t b}
$$


where

$$
\begin{aligned}
& \mathbf{X}^{t b}= \\
& {\left[\begin{array}{cccc}
1 & 0 & \cdots & \cdots \\
\left(-\frac{P}{\xi^{2}}-\frac{4 E I}{\xi^{4}}\right) & \left(\frac{\rho}{\eta^{2}}+\frac{2 P}{\xi^{2}}+\frac{7 E I}{\xi^{4}}\right) & \left(-\frac{P}{\xi^{2}}-\frac{4 E I}{\xi^{4}}\right) & \left(\frac{E I}{\xi^{4}}\right)
\end{array}\right.} \\
& \left(\frac{E I}{\xi^{4}}\right) \quad\left(-\frac{P}{\xi^{2}}-\frac{4 E I}{\xi^{4}}\right) \quad\left(\frac{\rho}{\eta^{2}}+\frac{2 P}{\xi^{2}}+\frac{6 E I}{\xi^{4}}\right) \quad\left(-\frac{P}{\xi^{2}}-\frac{4 E I}{\xi^{4}}\right) \\
& 0 \\
& \cdot \\
& \ddots \\
& \ddots \\
& \ddots \\
& \ddots \\
& 0 \\
& \left(\frac{E I}{\xi^{4}}\right) \\
& \text {.. } \\
& 0 \\
& 0 \\
& \text { L } \\
& \text {. } \\
& 0 \\
& \cdots \\
& \cdots \\
& \text {.. } \\
& \left(\frac{E I}{\xi^{4}}\right) \\
& 0 \\
& \ddots \quad \cdot \\
& \ddots \quad \cdot \cdot \quad \cdot \\
& \left(-\frac{P}{\xi^{2}}-\frac{4 E I}{\xi^{4}}\right) \quad\left(\frac{\rho}{\eta^{2}}+\frac{2 P}{\xi^{2}}+\frac{6 E I}{\xi^{4}}\right) \quad\left(-\frac{P}{\xi^{2}}-\frac{4 E I}{\xi^{4}}\right) \\
& \left(-\frac{E I}{\xi^{2}}-\frac{I_{m}}{2 \xi \eta^{2}}\right)\left(\begin{array}{c}
P+\frac{4 E I}{\xi^{2}} \\
+\frac{2 I_{m}}{\xi \eta^{2}}+\frac{P I_{m} \xi}{2 E I \eta^{2}}
\end{array}\right)\left(\begin{array}{c}
-\frac{\rho \xi^{2}}{\eta^{2}}-2 P \\
-\frac{5 E I}{\xi^{2}}-\frac{7 I_{m}}{2 \xi \eta^{2}} \\
-\frac{\rho I_{m} \xi^{3}}{2 E I \eta^{4}}-\frac{P I_{m} \xi}{E I \eta^{2}}
\end{array}\right) \\
& \left(\frac{E I}{\xi^{3}}\right) \quad\left(-\frac{P}{\xi}-\frac{3 E I}{\xi^{3}}\right) \quad\left(\frac{\rho \xi}{\eta^{2}}+\frac{P}{\xi}+\frac{3 E I}{\xi^{3}}\right) \quad\left(\frac{\rho \xi}{2 \eta^{2}}-\frac{E I}{\xi^{3}}+\frac{m}{\eta^{2}}+k\right)
\end{aligned}
$$




$$
\mathbf{Y}^{t b}=\left[\begin{array}{c}
0 \\
\frac{\rho}{\eta} v_{2}^{j-1}+\frac{\rho}{\eta^{2}} u_{2}^{j-1} \\
\vdots \\
\vdots \\
\vdots \\
\frac{\rho}{\eta} v_{I-2}^{j-1}+\frac{\rho}{\eta^{2}} u_{I-2}^{j-1} \\
M_{j}+\frac{I_{m}}{2 \xi \eta} v_{I+1}^{j-1}+\frac{I_{m}}{2 \xi \eta^{2}} u_{I+1}^{j-1} \\
-\left(\frac{\rho \xi^{2}}{\eta}+\frac{I_{m}}{2 \xi \eta}+\frac{\rho I_{m} \xi^{3}}{2 E I \eta^{3}}\right) v_{I-1}^{j-1} \\
-\left(\frac{\rho \xi^{2}}{\eta^{2}}+\frac{I_{m}}{2 \xi \eta^{2}}+\frac{\rho \rho I \xi^{3}}{2 E I \eta^{4}}\right) u_{I-1}^{j-1} \\
F_{j}+\frac{\rho \xi}{\eta} v_{I-1}^{j-1}+\frac{\rho \xi}{\eta^{2}} u_{I-1}^{j-1} \\
+\left(\frac{\rho \xi}{2 \eta}+\frac{m}{\eta}\right) v_{I}^{j-1}+\left(\frac{\rho \xi}{2 \eta^{2}}+\frac{m}{\eta^{2}}\right) u_{I}^{j-1}
\end{array}\right]
$$

Displacement and velocity vectors at the first time level are determined from initial conditions of the system. After obtaining displacements at the time level $j, u_{0}^{j}, u_{I+1}^{j}, u_{-1}^{j}$, and $u_{I+2}^{j}$ can be calculated from Eqs. (B.10)-(B.13), respectively, and the first, second, and third spatial derivatives of the displacements are calculated from Eqs. (A.5), (A.6), and (B.2) with $i=1, \cdots, I$, respectively.

\section{References}

[1] L. Meirovitch, 1997, Principles and Techniques of Vibrations, Prentice-Hall, Upper Saddle River, NJ.

[2] W.D. Zhu, and H. Ren, 2013, "An Accurate Spatial Discretization and Substructure Method with Application to Moving Elevator Cable-Car Systems - Part I: Methodology," ASME Journal of Vibration and Acoustics, 135(5), p. 051036.

[3] H. Ren, and W.D. Zhu, 2013, "An Accurate Spatial Discretization and Substructure Method with Application to Moving Elevator Cable-Car Systems - Part II: Application," ASME Journal of Vibration and Acoustics, 135(5), p. 051037.

[4] G.D. Smith, 1985, Numerical Solution of Partial Differential Equations: Finite Difference Methods, Oxford University Press Inc., New York, NY.

[5] P. Ŝln, 2005, Partial Differential Equations and the Finite Element Method, John Wiley \& Sons, Hoboken, NJ. 
[6] J.Y. Yeh, and L.W. Chen, 2006, "Wave Propagations of a Periodic Sandwich Beam by FEM and the Transfer Matrix Method," Composite Structures, 73(1), pp. 53-60.

[7] E. Kita, and N. Kamiya, 1995, "Trefftz Method: An Overview," Advances in Engineering Software, 24(1), pp. 3-12.

[8] C. Vanmaele, D. Vandepitte, and W. Desmet, 2007, "An Efficient Wave Based Prediction Technique for Plate Bending Vibrations," Computer Methods in Applied Mechanics and Engineering, 196(33), pp. 3178-3189.

[9] J.H. Ginsberg, 2001, Mechanical and Structural Vibrations, Wiley, New York, NY.

[10] J.C., Strikwerda, 2007, Finite Difference Schemes and Partial Differential Equations, Society for Industrial and Applied Mathematics, Philadelphia, pp. 17-64. 